\title{
Degenerations and limit Frobenius structures in rigid cohomology
}

\author{
Alan G. B. Lauder \\ In loving memory of my father George Alan Lauder 1937-2008.
}

\begin{abstract}
We introduce a 'limiting Frobenius structure' attached to any degeneration of projective varieties over a finite field of characteristic $p$ which satisfies a $p$-adic lifting assumption. Our limiting Frobenius structure is shown to be effectively computable in an appropriate sense for a degeneration of projective hypersurfaces. We conjecture that the limiting Frobenius structure relates to the rigid cohomology of a semistable limit of the degeneration through an analogue of the Clemens-Schmidt exact sequence. Our construction is illustrated, and conjecture supported, by a selection of explicit examples.
\end{abstract}

\section{Introduction}

This paper grew out of the author's attempt to generalise an algorithm for computing zeta functions of smooth projective hypersurfaces over finite fields to the case of singular hypersurfaces. This existing algorithm is called the deformation method, as the geometric idea behind the method is to deform a smooth diagonal hypersurface to the hypersurface in which one is interested. In this paper we degenerate smooth projective hypersurfaces to singular projective hypersurfaces, possibly not even reduced or irreducible, and try to compute some $p$-adic cohomological data attached to the degeneration. The problem we address is, how does one compute this cohomological data and what is its geometric meaning? Let us begin by sketching the original deformation method for smooth projective hypersurfaces $[\mathbf{1 0}, \mathbf{2 2}]$.

Let $\mathbb{F}_{q}$ be the finite field with $q$ elements of characteristic $p$, and

$$
P_{0} \in \mathbb{F}_{q}\left[x_{0}, x_{1}, \ldots, x_{n+1}\right]
$$

be a homogeneous polynomial of degree $d$ defining a smooth hypersurface $X_{0}$ in $\mathbb{P}_{\mathbb{F}_{q}}^{n+1}$. Recall that the zeta function of $X_{0}$ is the formal power series

$$
Z\left(X_{0}, T\right):=\exp \left(\sum_{k=1}^{\infty}\left|X_{0}\left(\mathbb{F}_{q^{k}}\right)\right| \frac{T^{k}}{k}\right)
$$

which encodes the number of points on $X_{0}$ over the different finite extensions $\mathbb{F}_{q^{k}}$ of the base field $\mathbb{F}_{q}$. By a famous theorem of Dwork, this series is the local expansion at the origin of a rational function with integer coefficients [8]. Define

$$
P_{1}:=x_{0}^{d}+x_{1}^{d}+\ldots+x_{n+1}^{d}
$$

and assume that $p$ does not divide $d$, so that the projective hypersurface $X_{1}$ defined by $P_{1}$ is smooth. Let $X \subset \mathbb{P}_{\mathbb{F}_{q}}^{n+1} \times \mathbb{A}_{\mathbb{F}_{q}}^{1}$ be the subvariety of smooth hypersurfaces in the pencil defined by the equation

$$
P_{t}:=(1-t) P_{0}+t P_{1}=0 .
$$

Received 28 December 2009; revised 6 September 2010.

2010 Mathematics Subject Classification 11G25 (primary), 11Y99, 14 D06 (secondary).

The author is a Royal Society University Research Fellow. His work is supported in part by a grant from the European Research Council (204083). 
Thus, there are an open curve $S \subseteq \mathbb{A}_{\mathbb{F}_{q}}^{1}$ and a smooth morphism $X \rightarrow S$ with generic fibre the smooth hypersurface defined by $P_{t}$. One can construct to any $p$-adic precision (in a welldefined sense) the relative rigid cohomology $\mathcal{H}:=H_{\text {rig }}^{n}(X / S)$ of the smooth projective family by starting with the cohomology $H_{\text {rig }}^{n}\left(X_{1}\right)$ of the smooth diagonal fibre and solving a $p$-adic differential equation. Since $X_{0}$ is also smooth, a base change theorem allows one to specialise $\mathcal{H}$ to an object $\mathcal{H}_{0}$ such that $\mathcal{H}_{0}=H_{\text {rig }}^{n}\left(X_{0}\right)$. Let $K$ be the unique unramified extension of the field of $p$-adic numbers $\mathbb{Q}_{p}$ with residue field $\mathbb{F}_{q}$, and $\sigma: K \rightarrow K$ the Frobenius automorphism. We shall not say anything in detail here about rigid cohomology, except that $H_{\text {rig }}^{n}\left(X_{0}\right)$ is a vector space of finite dimension over $K$ which is acted upon by a $\sigma$-linear map $\mathcal{F}_{0}$ called Frobenius. The trace formula in rigid cohomology

$$
Z\left(X_{0}, T\right)=\frac{\operatorname{det}\left(1-T \mathcal{F}_{0}^{\log _{p}(q)} \mid H_{\text {rig }}^{n}\left(X_{0}\right)\right)^{(-1)^{n+1}}}{(1-T)(1-q T)\left(1-q^{2} T\right) \ldots\left(1-q^{n} T\right)}
$$

now allows us to recover the zeta function exactly, assuming that we have carried enough $p$-adic precision in our computation.

When $X_{0}$ is singular, the above algorithm fails, in both theory and practice. One cannot specialise $\mathcal{H}=H_{\text {rig }}^{m}(X / S)$ at the point $t=0$ given the manner in which it is constructed in our original method, and even if one could there is no base change theorem to tell you what this gives.

In this paper we consider the case in which $X_{0}$ is (almost) any projective hypersurface, perhaps singular or reducible, or even not reduced as a scheme. In fact, we work in slightly greater generality than above, but subject to one lifting condition. We consider a smooth family $X \rightarrow S \subseteq \mathbb{A}_{\mathbb{F}_{q}}^{1}$ of projective hypersurfaces of degree $d$ and dimension $n$ which lifts to a smooth family $\mathcal{X}_{\mathcal{O}_{L}} \rightarrow \mathcal{S}_{\mathcal{O}_{L}}$ of projective hypersurfaces of degree $d$ over the ring of integers $\mathcal{O}_{L}$ of an algebraic number field $L \subset K$ such that all fibres in the punctured $p$-adic open unit disk around the origin are 'smooth and in general position' (Definition 2.7). It is convenient to further assume $1 \in S$. We say then that $X \rightarrow S$ is in general position and has a good algebraic lift around $t=0$ (Definition 3.14). Defining as before $\mathcal{H}:=H_{\text {rig }}^{n}(X / S)$, we now introduce a new object $\left(\mathcal{H}_{0}, \mathcal{N}_{0}, \mathcal{F}_{0}, e\right)$, which we call the limiting (mixed) Frobenius structure at $t=0$. It is a finite-dimensional vector space $\mathcal{H}_{0}$ over the $p$-adic field $K$ with an invertible $\sigma$-linear operator $\mathcal{F}_{0}: \mathcal{H}_{0} \rightarrow \mathcal{H}_{0}$ and a nilpotent operator $\mathcal{N}_{0}: \mathcal{H}_{0} \rightarrow \mathcal{H}_{0}$ with $\mathcal{N}_{0}^{n+1}=0$ such that

$$
\mathcal{N}_{0} \mathcal{F}_{0}=p \mathcal{F}_{0} \mathcal{N}_{0}
$$

and in addition a positive integer $e$. We call $\mathcal{F}_{0}$ the Frobenius operator and $\mathcal{N}_{0}$ the monodromy operator. The integer $e$ specifies a cover $S_{e} \rightarrow S\left(t \mapsto t^{e}\right)$ of the base curve which occurs during the construction. The monodromy operator $\mathcal{N}_{0}$ is defined over the algebraic number field $L \subset K$ and so we may represent it explicitly via a nilpotent matrix of algebraic numbers with respect to some basis of $\mathcal{H}_{0}$. With respect to the same basis, the matrix for the Frobenius $\mathcal{F}_{0}$ has entries in the uncountable field $K$ and one cannot write it down; however, for each positive integer $N$ one may approximate it by a matrix $\tilde{\mathcal{F}}_{0}$ with entries in the algebraic number field $L$ such that $\operatorname{ord}_{p}\left(\mathcal{F}_{0}-\tilde{\mathcal{F}}_{0}\right) \geqslant N$. We call such a $\tilde{\mathcal{F}}_{0}$ a $p^{N}$-approximation to the matrix for $\mathcal{F}_{0}$. Our main theoretical result is as follows (Theorem 3.15).

Theorem 1.1. The limiting Frobenius structure $\left(\mathcal{H}_{0}, \mathcal{N}_{0}, \mathcal{F}_{0}, e\right)$ at $t=0$ for a degeneration of smooth projective hypersurfaces $X \rightarrow S$ which has a good algebraic lift around $t=0$ and is in general position is effectively computable, in the following sense. Given such a degeneration and any positive integer $N$, one may compute $e$, a basis for $\mathcal{H}_{0}$, and with respect to this basis the matrix for $\mathcal{N}_{0}$ and a $p^{N}$-approximation to a matrix for $\mathcal{F}_{0}$.

Our algorithm for computing the limiting Frobenius structure is deterministic and explicit. It is practical in two senses. First, one may show that it is polynomial-time in $p, \log _{p}(q),(d+1)^{n}$ and $N$ under favourable assumptions (§3.3.2). Second, the author has implemented the 
algorithm in the programming language MAGMA, and executed it for degenerations of curves, surfaces and threefolds of various different degrees (Section 5).

Given that one can define and compute the limiting Frobenius structure, it is natural to next ask for its geometric significance. When $0 \in S$, the fibre $X_{0}$ in $X \rightarrow S$ is smooth. In this case, $e=1, \mathcal{N}_{0}=0$ and $\mathcal{F}_{0}$ is the usual Frobenius map on $\mathcal{H}_{0}=H_{\text {rig }}^{n}\left(X_{0}\right)$. Let us suppose then that $0 \notin S$ and so $X_{0}$ is not defined. One may canonically extend $X \rightarrow S$ to a flat family $Y \rightarrow T$ of projective hypersurfaces over $T:=S \cup\{0\}$. Let us denote the fibre over the origin in $Y \rightarrow T$ as $X_{0}$ and call it the degenerate fibre. One sees from some examples (Examples 5.2, 5.3, 5.8 and 5.13) that there may be no relationship between the limiting Frobenius structure and the zeta function of $X_{0}$. Before proceeding further, we need some more definitions.

Let us denote by $S_{e} \rightarrow S$ and $T_{e} \rightarrow T$ the covers defined by the map $t \mapsto t^{e}$, and write $X_{e}:=X \times{ }_{S} S_{e}$. (It is a consequence of our lifting hypothesis that the integer $e$ is always coprime to $p$ (Note 3.9), and so the cover $S_{e} \rightarrow S$ is unramified.) Note that $X_{e} \rightarrow S_{e}$ is still a smooth family of projective hypersurfaces. We shall say that $X_{e} \rightarrow S_{e}$ extends to a semistable degeneration over the origin if the following is true. There exist a smooth $\mathbb{F}_{q}$-variety $Y_{e}$ and a flat morphism $Y_{e} \rightarrow T_{e}$ which restricts over $S_{e} \subset T_{e}$ to $X_{e} \rightarrow S_{e}$ with the fibre $Y_{e, 0}$ over $0 \in T$ a reduced union of smooth hypersurfaces on $Y_{e}$ crossing transversally. When $X_{e} \rightarrow S_{e}$ extends to a semistable degeneration $Y_{e} \rightarrow T_{e}$ over the origin, we shall sometimes denote the degenerate fibre $Y_{e, 0}$ by $X_{e, 0}$, bearing in mind always that it depends upon the choice of extension. We make the following conjecture, motivated in part by an analogy with degenerations of complex algebraic varieties (Theorem 4.3). (See Conjecture 4.11 for a fuller statement.)

Conjecture 1.2. The morphism $X_{e} \rightarrow S_{e}$ extends to a semistable degeneration over the origin with degenerate fibre $X_{e, 0}$. Moreover, for any such extension there exists a 'Clemens-Schmidt' exact sequence in rigid cohomology containing the following:

$$
\ldots \rightarrow H_{\text {rig }}^{n}\left(X_{e, 0}\right) \rightarrow \mathcal{H}_{0} \stackrel{\mathcal{N}_{0}}{\longrightarrow} \mathcal{H}_{0}(-1) \rightarrow \ldots
$$

The exactness of the above three-term sequence in the middle ('local invariant cycle theorem') implies that $\operatorname{det}\left(1-T \mathcal{F}_{0}^{\log _{p}(q)} \mid \operatorname{Ker}\left(\mathcal{N}_{0}\right)\right)$ occurs as a factor in the 'middle Weil polynomial' of the zeta function of the semistable variety $X_{e, 0}$. The main experimental work in this paper is the testing of our conjecture for some degenerations of low-dimensional hypersurfaces (Section 5).

Having shown that the limiting Frobenius structure for a degeneration of hypersurfaces under a lifting assumption is effectively computable, and provided some experimental evidence supporting a conjectural geometric interpretation of this structure, next one might ask if all this is of any use. Let us call the algorithm in this paper the degeneration method. The author suggests at least two practical applications of this method.

When the morphism $Y \rightarrow T$ canonically extending $X \rightarrow S$ over the origin is semistable, under Conjecture 4.11 the degeneration method computes cohomological information about $X_{0}$ itself. In particular, the algorithm can (conjecturally) be used to compute the zeta function of a nodal plane projective curve, by embedding it as the degenerate fibre in a suitable family. It is a classical fact that any irreducible projective curve over an algebraically closed field is birational to a plane projective curve with only nodes as singularities [19, Theorem 1.61], and this transformation is effective. Thus, given any geometrically irreducible projective curve over $\mathbb{F}_{q}$, one can explicitly find a semistable plane model over an extension of $\mathbb{F}_{q}$, and use the degeneration method to compute the zeta function of this plane model; see Example 5.7.

For a second application of the degeneration method, recall that irreducible plane curves with a single node arise naturally when one is interested in calculating the L-series of a smooth plane curve defined over the integers. The cohomology of these curves gives the factors at the bad primes. The degeneration method will compute these factors, under Conjecture 4.11; see Note 5.6 for an example. 
We make two concluding remarks. First, the existence of a suitably defined limiting Frobenius structure at $t=0$ for any smooth projective family $X \rightarrow S \subset \mathbb{A}_{\mathbb{F}_{q}}^{1} \backslash\{0\}$ of varieties actually follows from the semistable reduction theorem of Kedlaya for overconvergent $F$-isocrystals on an open curve [16]; indeed, directly from its local incarnation the $p$-adic local monodromy theorem. We have just given an independent and effective proof of its existence for the case of hypersurfaces subject to a lifting assumption, and given the object a name; see Note 3.17 for a discussion of nomenclature. (In fact, we show its existence for any smooth proper family $X \rightarrow S$ subject to a lifting assumption (Definition 3.10 and Theorem 3.8); but without the lifting assumption the situation is much more difficult, and one needs to allow different covers of $S$ than just $t \mapsto t^{e}$.) Kedlaya's semistable reduction theorem motivated the author to try to adapt the deformation method to handle degenerations.

Second, there is a close analogy with the theory of degenerations of smooth complex algebraic varieties where one has a limiting mixed Hodge structure [32, Chapter 11]. However, our situation is even richer. Section 3.4 sketches the construction of a limiting (mixed) Frobenius-Hodge structure at $t=0$ in dimension $m$ attached to a smooth and proper morphism $\mathcal{X}_{\mathcal{O}_{L}} \rightarrow \mathcal{S}_{\mathcal{O}_{L}} \subset \mathbb{A}_{\mathcal{S}_{\mathcal{O}_{L}}}^{1}$ and a suitably large rational prime $p$ inert in the ring of integers $\mathcal{O}_{L}$ of a number field $L$. This object is new to the author. It is a quadruple $\left(\mathcal{H}_{0}^{\bullet}, \mathcal{N}_{0}, \mathcal{F}_{0}, e\right)_{W}$ consisting of a filtered $W$-module $\mathcal{H}_{0}^{\bullet}$ with operators $\mathcal{N}_{0}$ and $\mathcal{F}_{0}$ such that $\mathcal{N}_{0} \mathcal{F}_{0}=p \mathcal{F}_{0} \mathcal{N}_{0}$, $\mathcal{N}_{0}^{m+1}=0$ and $\mathcal{F}_{0} \mathcal{H}_{0}^{i} \subseteq p^{i} \mathcal{H}_{0}$, along with a positive integer $e$. (Here $W$ is the ring of integers of the $p$-adic field $K$.) When $e=1$, the pair ' $\left(\mathcal{H}_{0}^{\bullet}, \mathcal{N}_{0}\right)_{W} \otimes_{W} \mathbb{C}^{\prime}$ is the usual limiting mixed Hodge structure at $t=0$ of the degeneration of complex algebraic varieties, and a similar triple to $\left(\mathcal{H}_{0}, \mathcal{N}_{0}, \mathcal{F}_{0}\right)_{W}$ occurs in crystalline cohomology (Hyodo-Kato cohomology in equicharacteristic). This structure is computable in an appropriate sense for a degeneration of hypersurfaces and suitably large prime $p$. One can then extend Conjecture 4.11 by insisting that everything is defined over $W$ and there is an exact sequence of 'Frobenius-Hodge structures'. The existence of this $W$-lattice structure and Hodge filtration on the limiting Frobenius structures informed the author's choice of precisions in the computations in Section 5.

The paper is organised in the following manner. Section 2 considers the algebraic de Rham cohomology of degenerations of varieties over a computable field of characteristic zero. The first main result is some kind of partly 'effective' semistable reduction theorem in algebraic de Rham cohomology (Theorem 2.4). We then focus on degenerations of projective hypersurfaces and give an entirely effective proof of semistable reduction for algebraic de Rham cohomology in this situation (Theorem 2.15). Section 3 considers degenerations in positive characteristic and introduces limiting Frobenius structures. After showing that the notion is well defined (§ 3.2.2), we use Theorem 2.4 to show that they exist under a lifting assumption (Theorem 3.8 and Definition 3.10). We then apply the theory of $\S 2.3$ to show that limiting Frobenius structures are effectively computable in an appropriate sense for degenerations of hypersurfaces subject to a lifting assumption (Definition 3.14 and Theorem 3.15). Next follows a brief sketch of how one endows our limiting Frobenius structures with an integral structure and Hodge filtration (§ 3.4). Section 4 presents our conjectural 'Clemens-Schmidt' exact sequence in rigid cohomology (Conjecture 4.11). Finally, in Section 5 we present an assortment of examples computed by the author which give some support to Conjecture 4.11 and also illustrate our constructions. Although the remaining sections are arranged in their logical order, we recommend that the reader begins by looking through Section 5 and then gently reverses through the paper before starting in earnest.

\section{Degenerations in characteristic zero}

Let $F$ be a field of characteristic zero. We wish to be able to compute with elements of $F$, so let us assume that $F$ is countable and one can effectively perform arithmetic operations in $F$. 


\subsection{Relative algebraic de Rham cohomology}

We refer to [14] for the definition of relative algebraic de Rham cohomology and the Gauss-Manin connection.

Let $S \subseteq \mathbb{A}_{F}^{1}$ be an open curve, and $X \rightarrow S$ be a smooth morphism of algebraic varieties over $F$ of relative dimension $n$. Fix an integer $m$ with $0 \leqslant m \leqslant 2 n$ and denote by $H_{d R}^{m}(X / S)$ the $m$ th relative algebraic de Rham cohomology group of $X \rightarrow S$.

Write $S=\operatorname{Spec}(A)$, where $A=F[t, 1 / \Delta(t)]$ for some polynomial $\Delta(t) \in F[t]$. Then $H_{d R}^{m}(X / S)$ is a locally free $A$-module of finite rank, which we may assume to be free since $A$ is a principal ideal domain (see for example [21, Chapter III.7]). Let

$$
\nabla_{\frac{d}{d t}}: H_{d R}^{m}(X / S) \stackrel{\nabla_{G M}}{\longrightarrow} H_{d R}^{m}(X / S) \otimes \Omega_{A}^{1} \stackrel{\mathrm{id} \otimes \frac{d}{d t}}{\longrightarrow} H_{d R}^{m}(X / S)
$$

denote the Gauss-Manin connection $\nabla_{G M}$ contracted with $\frac{d}{d t} \in \operatorname{Hom}\left(\Omega_{A}^{1}, A\right)$. To ease notation, we shall write $\nabla:=\nabla_{\frac{d}{d t}}$ and call this the Gauss-Manin connection. The connection $\nabla$ is Leibniz linear; that is,

$$
\begin{gathered}
\nabla(r+s)=\nabla(r)+\nabla(s) \\
\nabla(a r)=\frac{d a}{d t} r+a \nabla(r)
\end{gathered}
$$

for $a \in A$ and $r, s \in H_{d R}^{m}(X / S)$.

\subsection{Regularity and semistable reduction}

Let $r$ be the rank of $H_{d R}^{m}(X / S)$ and $\mathcal{B}$ be a basis. Denote by $N(t)$ the matrix for the GaussManin connection acting on this basis. The matrix $N(t)$ has entries in $A$. If one changes basis by a matrix $H \in \mathrm{GL}_{r}(A)$, then, since $\nabla$ is Leibniz linear, the new matrix for $\nabla$ with respect to the basis $\mathcal{B}_{[H]}:=\{H b\}_{b \in \mathcal{B}}$ is

$$
N_{[H]}:=H N H^{-1}+\frac{d H}{d t} H^{-1} .
$$

Suppose $\Delta(0)=0$, so that $N(t)$ may have a pole at $t=0$. Then the 'regularity theorem' guarantees that there exists a matrix $H \in \mathrm{GL}_{r}(F((t)))$ such that $N_{[H]}$ has only a simple pole at $t=0$; see for example [13]. We use this theorem to prove a stronger and effective result.

Theorem 2.1 (Regularity). There exists an explicit deterministic algorithm with the following input and output. The input is the matrix $N(t)$ for the Gauss-Manin connection $\nabla$ on $H_{d R}^{m}(X / S)$ with respect to some basis $\mathcal{B}$, where $X \rightarrow S$ is a smooth morphism of varieties over a computable field $F$. The output is a matrix $H \in \mathrm{GL}_{r}\left(F\left[t, t^{-1}\right]\right)$ such that the matrix $N_{[H]}$ for $\nabla$ with respect to the basis $\mathcal{B}_{[H]}$ of $H_{d R}^{m}(X / S)$ has only a simple pole at $t=0$.

Proof. If $\Delta(0) \neq 0$, then we are done since the matrix $N(t)$ will not have a pole at $t=0$. So, assume $\Delta(0)=0$. For $\omega \in H_{d R}^{m}(X / S)$, let $\mathcal{B}_{\omega}:=\left\{(t \nabla)^{i}(\omega)\right\}_{0 \leqslant i<r}$. By definition, $\omega$ is a cyclic vector for the differential system $\left(H_{d R}^{m}(X / S),(t \nabla)\right)$ over $F((t))$ if and only if $\mathcal{B}_{\omega}$ is a linearly independent set over $F((t))$. For any given $\omega$ expressed in the basis $\mathcal{B}$, one may check whether $\omega$ is cyclic by computing a determinant, since one can calculate the action of $t \nabla$ via $t \frac{d}{d t}+t N(t)$. Now a cyclic vector exists amongst a certain finite list of $F[t]$-linear combinations of the basis $\mathcal{B}$ according to [34, Lemma 2.10]. Thus, one may effectively find a cyclic vector, $\omega$ say. The differential system is regular, by the regularity theorem. Thus, by [17, Corollary 7.1.3], the matrix for the connection with respect to the basis $\mathcal{B}_{\omega}$ of $H_{d R}^{m}(X / S) \otimes_{A} F((t))$ has only a simple pole at $t=0$. We can compute the change of basis matrix $G$ between $\mathcal{B}$ and $\mathcal{B}_{\omega}$, since we can explicitly compute the action of $(t \nabla)^{i}$ on $\omega$. Note that $G \in \mathrm{GL}_{r}(F(t))$. Embedding $F(t) \hookrightarrow F((t))$ and using Lemma 2.2, we may factor $G=L H$, where $H \in \mathrm{GL}_{r}\left(F\left[t, t^{-1}\right]\right)$ and 
$L \in \mathrm{GL}_{r}(F[[t]])$. Then $N_{[H]}$ has only a simple pole at $t=0$, since $N_{[H]}=\left(N_{[G]}\right)_{\left[L^{-1}\right]}$ with $N_{[G]}$ having a simple pole and $L^{-1}$ no pole at $t=0$. Moreover, $\mathcal{B}_{[H]}$ is a global basis for $H_{d R}^{m}(X / S)$, since $H(t) \in \mathrm{GL}_{r}\left(F\left[t, t^{-1}\right]\right) \subseteq \mathrm{GL}_{r}(A)$. Thus, $H$ is the desired change of basis matrix.

Lemma 2.2. Given any $G \in \mathrm{GL}_{r}(F((t)))$, there exist $H \in \mathrm{GL}_{r}\left(F\left[t, t^{-1}\right]\right)$ and $L \in$ $\mathrm{GL}_{r}(F[[t]])$ such that $G=L H$. Moreover, if $G \in \mathrm{GL}_{r}(F(t)) \subset \mathrm{GL}_{r}(F((t)))$, then such $H$ and $L \in \mathrm{GL}_{r}(F[[t]] \cap F(t))$ are effectively computable.

Proof. Let $G \in \mathrm{GL}_{r}(F((t)))$. We prove by induction on $r$ that there exists $L \in \mathrm{GL}_{r}(F[[t]])$ such that $L G$ is upper triangular with monomials on the diagonal and elements in $F\left[t, t^{-1}\right]$ above the diagonal. The case $r=1$ is immediate. For $r>1$, by row operations (equivalently, premultiplying by a suitable matrix in $\left.\mathrm{GL}_{r}(F[[t]])\right)$ one may reduce to the case in which $G$ is upper triangular with monomials on the diagonal and elements in $F((t))$ above the diagonal. Next, by induction one may assume that all entries in $G$ above the diagonal except those on the first row lie in $F\left[t, t^{-1}\right]$. Finally, by an explicit calculation, one shows that there exists a matrix $L \in \mathrm{GL}_{r}(F[[t]])$ of the following form such that $L G$ is as required: $L$ equals the identity matrix except for elements in positions $(1, i)$ for $2 \leqslant i \leqslant r$. Note that the factorisation is unique up to multiplication by an element of $\mathrm{GL}_{r}(F[t])$. In the case in which $G \in \mathrm{GL}_{r}(F(t))$, one checks that this proof describes an effective algorithm and $L \in \mathrm{GL}_{r}(F[[t]] \cap F(t))$.

Note 2.3. The use of a cyclic vector and the factorisation in Lemma 2.2 were suggested to the author by Kiran Kedlaya. Kedlaya also suggests replacing the use of a cyclic vector by a basis over $F[[t]]$ for the $(t \nabla)$-stable lattice in $F((t))$ generated by the elements $\left\{(t \nabla)^{i}(b)\right\}$ for $0 \leqslant i<r$ and $b \in \mathcal{B}$. We further note that one could use Katz's explicit construction of a cyclic vector [34, Lemma 2.11]; see paragraph 2 in the proof of [17, Theorem 5.4.2] for how to get around the assumption of Katz that one has an element ' $z$ ' in $F((t))$ with ' $z$ ' $=1$ ', where in our case the dash is the operator $t \frac{d}{d t}$.

The significance of Theorem 2.1 is that using a change of basis matrix which is invertible over $F\left[t, t^{-1}\right]$ does not introduce any new poles in the matrix for the connection. Thus, when $F$ is a countable subfield of the field of $p$-adic numbers (or the complex numbers), one does not change the radius of convergence on the punctured disk around zero of the connection matrix. This is essential in applications of the theorem to $p$-adic cohomology (Section 3).

We now turn to the problem of semistable reduction. For $e$ a positive integer, write $A_{e}:=F\left[s, 1 / \Delta\left(s^{e}\right)\right]$ and $S_{e}:=\operatorname{Spec}\left(A_{e}\right)$. Define $A \rightarrow A_{e}$ by $t \mapsto s^{e}$ to get a morphism $S_{e} \rightarrow S$. One may pull back the family $X \rightarrow S$ via $S_{e} \rightarrow S$ to get $X_{e} \rightarrow S_{e}$, where $X_{e}:=X \times{ }_{S} S_{e}$. We have

$$
H_{d R}^{m}\left(X_{e} / S_{e}\right) \cong H_{d R}^{m}(X / S) \otimes_{A} A_{e},
$$

since $A_{e}$ is a flat $A$-module. For a basis $\mathcal{B}$ of $H_{d R}^{m}(X / S)$, let $\mathcal{B}_{e}:=\left\{b \otimes 1_{A_{e}}\right\}$ be the associated basis of $H_{d R}^{m}\left(X_{e} / S_{e}\right)$. As before, let $N(t)$ be the matrix for the Gauss-Manin connection with respect to the basis $\mathcal{B}$. Then the matrix for the Gauss-Manin connection

$$
\nabla_{e}: H_{d R}^{m}\left(X_{e} / S_{e}\right) \stackrel{\nabla_{G M}}{\longrightarrow} H_{d R}^{m}\left(X_{e} / S_{e}\right) \otimes \Omega_{A_{e}}^{1} \stackrel{\text { id } \otimes \frac{d}{d s}}{\longrightarrow} H_{d R}^{m}\left(X_{e} / S_{e}\right)
$$

with respect to the basis $\mathcal{B}_{e}$ is

$$
\left(e s^{e-1}\right) N\left(s^{e}\right)
$$

since $d t=e s^{e-1} d s$.

The problem of local semistable reduction in algebraic de Rham cohomology is to find a positive integer $e$ and a basis for $H_{d R}^{m}\left(X_{e} / S_{e}\right)$ such that the matrix for the connection $\nabla$ has only a simple pole at $s=0$ with nilpotent residue matrix. 
TheOREm 2.4 (Semistable reduction). There exists an explicit deterministic algorithm with the following input and output. The input is the matrix $N(t)$ for the Gauss-Manin connection on $H_{d R}^{m}(X / S)$ with respect to some basis $\mathcal{B}$, where $X \rightarrow S$ is a smooth morphism of varieties over a computable field $F$. The output is an integer $e$ and a matrix $H(s) \in \mathrm{GL}_{r}\left(F\left[s, s^{-1}\right]\right)$ such that the matrix

$$
\left(e s^{e-1} N\left(s^{e}\right)\right)_{[H(s)]}:=H e s^{e-1} N\left(s^{e}\right) H^{-1}+\frac{d H}{d s} H^{-1}
$$

for the Gauss-Manin connection $\nabla_{e}$ on the basis $\left(\mathcal{B}_{e}\right)_{[H(s)]}$ of $H_{d R}^{m}\left(X_{e} / S_{e}\right)$ has only a simple pole at $s=0$ with nilpotent residue matrix.

Proof. By Theorem 2.1, one reduces to the case in which $N(t)$ has only a simple pole at $t=0$. Let $\left\{\lambda_{i}\right\}_{1 \leqslant i \leqslant r}$ denote the eigenvalues of the residue matrix. By the local monodromy theorem, we have $\lambda_{i} \in \mathbb{Q}$; see [13]. Thus, we may find a smallest positive integer $e$ such that $e \lambda_{i} \in \mathbb{Z}$ for all $1 \leqslant i \leqslant r$. Then $e s^{e-1} N\left(s^{e}\right)$ has only a simple pole at $s=0$ and a residue matrix with integer eigenvalues. Shearing transformations now allow one to find a matrix $H(s) \in \mathrm{GL}_{r}\left(F\left[s, s^{-1}\right]\right)$ such that $\left(e s^{e-1} N\left(s^{e}\right)\right)_{[H]}$ has only a simple pole with nilpotent residue matrix [9, Lemma III.8.2]. (Note that since in our application the eigenvalues of the residue matrix lie in $\mathbb{Z}$, the initial reduction to upper triangular form of the residue matrix in the proof of [9, Lemma III.8.2] can be performed over F.)

Note 2.5. Since the change of basis matrix $H$ lies in $\operatorname{GL}_{r}\left(F\left[s, s^{-1}\right]\right)$, one can perform global semistable reduction over the whole of the affine basis $S$. That is, after pulling back by a chain of unramified covers $S_{e} \rightarrow S$, effectively find a matrix for the connection which has at worst simple poles at all finite points with nilpotent residue matrices. Moreover, this can be done without introducing any new finite poles to the differential system. Alternatively, without a base change one can effectively find a matrix for the connection with only simple poles at all finite points - this is useful when computing in the cokernel

$$
H_{d R}^{1}\left(S, H_{d R}^{m}(X / S)\right):=\operatorname{Coker}\left(\nabla_{G M}\right)
$$

of the connection $\nabla_{G M}[\mathbf{2 3}$, Section 4].

\subsection{Hypersurfaces}

The first significant result of this section is Theorem 2.13, on the construction of the PicardFuchs differential system for families of projective hypersurfaces. Our construction is based upon the method of Griffiths and Dwork. However, it differs from the usual presentation in the literature (for example $[\mathbf{5}, \S 5.3]$ ) in one key way. We avoid Gröbner basis computations, and instead work with linear algebra and Macaulay-style resultant matrices. This has a number of benefits. First, it allows us to give an explicit a priori characterisation of the locus of poles of the Picard-Fuchs system with respect to a particular basis, and thus prove a theorem on explicit semistable reduction for hypersurfaces (Theorem 2.15). Second, it reveals additional structure in the Picard-Fuchs system (Corollary 2.14). Third, in the author's own experience, it is faster in practical implementations (Note 2.9). Combining Theorems 2.4 and 2.13 yields Theorem 2.15, the main result of Section 2.

2.3.1. Cohomology of the generic hypersurface. In this section, we fix $n \geqslant 0$ and $d \geqslant 1$ and let $x_{0}, x_{1}, \ldots, x_{n+1}$ be variables. For $w=\left(w_{0}, w_{1}, \ldots, w_{n+1}\right) \in \mathbb{Z}_{\geqslant 0}^{n+2}$, define $x^{w}:=$ $x_{0}^{w_{0}} x_{1}^{w_{1}} \ldots x_{n+1}^{w_{n+1}}$ and $|w|:=w_{0}+w_{1}+\ldots+w_{n+1}$. For each $w \in \mathbb{Z}_{\geqslant 0}^{n+2}$ with $|w|=d$, we introduce a variable $a_{w}$ and define $P:=\sum_{|w|=d} a_{w} x^{w}$ to be the 'generic polynomial' homogeneous of degree $d$ with coefficients in the multivariate polynomial ring $R:=\mathbb{Z}\left[a_{w}|| w \mid=d\right]$. 
Write

$$
\Omega:=\sum_{i=0}^{n+1}(-1)^{i} x_{i} d x_{0} \wedge \ldots \wedge \widehat{d x}_{i} \wedge \ldots \wedge d x_{n+1},
$$

where the hat denotes omission, and define

$$
\mathcal{B}_{U}:=\left\{\frac{x^{w} \Omega}{P^{k}} \mid 0 \leqslant w_{i}<d-1 \text { and } k \in \mathbb{Z} \text { with }|w|+n+2=k d\right\} .
$$

Elements in $\mathcal{B}_{U}$ are differential forms on a certain affine scheme $U$, which is defined in the statement of the following theorem.

THEOREM 2.6. There exists an explicitly computable non-zero polynomial $\Delta_{n, d} \in R$ such that the following is true. Define $R^{(1)}:=R\left[1 / \Delta_{n, d}\right]$ and $T:=\operatorname{Spec}\left(R^{(1)}\right)$. Let $X \subset \mathbb{P}_{T}^{n+1}$ be the projective hypersurface defined by the polynomial $P$, and let $U$ be the affine complement of $X$ in $\mathbb{P}_{T}^{n+1}$. Then the set of cohomology classes $\left\{[b] \mid b \in \mathcal{B}_{U}\right\} \subset H_{d R}^{n+1}(U / T)$ is an $R^{(1)}$-basis for $H_{d R}^{n+1}(U / T)$.

Proof. This is an explicit computation carried out in $\S 2.3 .2$.

Definition 2.7. We shall say that a hypersurface of degree $d$ and dimension $n$ over a field of characteristic zero is smooth and in general position if its coefficients are not zeros of $\Delta_{n, d}$.

It will follow immediately from our definition of $\Delta_{n, d}$ in $\S 2.3 .2$ that a hypersurface which is 'smooth and in general position' is indeed smooth.

With the notation of the theorem above, there is an algebraically defined map called the residue map:

$$
H_{d R}^{n+1}(U / T) \stackrel{\text { Res }}{\longrightarrow} H_{d R}^{n}(X / T) .
$$

It is injective and the image is by (our) definition the primitive middle-dimensional cohomology

$$
\mathcal{H}_{T}:=\operatorname{Prim} H_{d R}^{n}(X / T) .
$$

Note that $\mathcal{H}_{T}=H_{d R}^{n}(X / T)$ when $n$ is odd, and $H_{d R}^{n}(X / T) / \mathcal{H}_{T}$ is free of rank one when $n$ is even. Define

$$
\mathcal{B}_{X}:=\left\{\operatorname{Res}([b]): b \in \mathcal{B}_{U}\right\}
$$

Corollary 2.8. With the notation of Theorem 2.6, the set of residues $\mathcal{B}_{X}$ is an $R^{(1)}$-basis for the primitive middle-dimensional algebraic de Rham cohomology $\mathcal{H}_{T}$ of $X \rightarrow T$.

We shall call $\mathcal{B}_{X}$ the Dwork basis of $\mathcal{H}_{T}$.

2.3.2. From the universal to the generic hypersurface. This section contains our explicit proof of Theorem 2.6. We examine the primitive middle-dimensional algebraic de Rham cohomology of a hypersurface, starting with a universal one and then specialising to the generic smooth hypersurface and then the hypersurface which is 'smooth and in general position'. Here 'in general position' is a utilitarian definition which allows us to write down a certain basis for cohomology.

Let us introduce some new notation for use only in this section. For the ring $R=\mathbb{Z}\left[a_{w}|| w \mid\right.$ $=d]$, let $\mathbb{P}_{R}^{n+1}$ denote projective space of dimension $n+1$ over $R$. Let $X / R$ be the 'universal' projective hypersurface of degree $d$ in $\mathbb{P}_{R}^{n+1}$ defined by the polynomial $P=\sum_{|w|=d} a_{w} x^{w}$. Denote by $U / R$ its affine complement. Then

$$
H_{d R}^{\bullet}(U / R):=\mathbb{H}\left(\Omega_{U / R}^{\bullet}\right)=H\left(\Gamma\left(U / R, \Omega_{U / R}^{\bullet}\right)\right) .
$$


Here $\mathbb{H}(\cdot)$ denotes hypercohomology of a complex of sheaves, $H(\cdot)$ homology of a complex of modules, and the second equality follows since $U / R$ is affine and so the Cech-de Rham spectral sequence for computing hypercohomology degenerates at the first term. In particular, since $\Omega_{U / R}^{n+2}=0$,

$$
H_{d R}^{n+1}(U / R)=\frac{\Gamma\left(U / R, \Omega_{U / R}^{n+1}\right)}{d \Gamma\left(U / R, \Omega_{U / R}^{n}\right)},
$$

where $d$ is derivation of $n$-forms. That is, $H_{d R}^{n+1}(U / R)$ is global (closed) $(n+1)$-forms modulo exact ones.

Here is an explicit description of $H_{d R}^{n+1}(U / R)$ - this is due to Griffiths but a convenient reference is $[\mathbf{1}$, Section 3]. For $k \geqslant 1$, define

$$
Z_{k}:=\left\{\frac{A \Omega}{P^{k}} \mid A \in R\left[x_{0}, \ldots, x_{n+1}\right] \text { homogeneous degree } k d-n-2\right\} .
$$

Then $Z_{k}$ is the $R$-module of $(n+1)$-forms on $\mathbb{P}_{R}^{n+1}$ defined on $U / R$ with poles of order at most $k$ along $X / R$; that is,

$$
Z_{k}=\Gamma\left(\mathbb{P}_{R}^{n+1}, \Omega_{\mathbb{P}_{R}^{n+1}}^{n+1}(k(X / R))\right) .
$$

Note $Z_{k} \subset Z_{k+1}$ for $k \geqslant 1$. Defining $Z:=\bigcup_{k=1}^{\infty} Z_{k}$, we have $Z=\Gamma\left(U / R, \Omega_{U / R}^{n+1}\right)$ with the natural filtration by pole order.

Let $b_{1}=\{0\}$ and for $k \geqslant 2$ define $b_{k}$ to be the $R$-module generated by

$$
\frac{\left(\delta_{i} A\right) \Omega}{P^{k-1}}-(k-1) \frac{A\left(\delta_{i} P\right) \Omega}{P^{k}}
$$

for all $A \in R\left[x_{0}, \ldots, x_{n+1}\right]$ homogeneous of degree $(k-1) d-n-1$ and all $0 \leqslant i \leqslant n+1$. Here $\delta_{i}:=\frac{\partial}{\partial x_{i}}$. Let $B_{k}$ be the $R$-module generated by $\bigcup_{j=1}^{k} b_{j}$. Then

$$
B_{k}=d \Gamma\left(\mathbb{P}_{R}^{n}, \Omega_{\mathbb{P}_{R}^{n+1}}^{n}((k-1)(X / R))\right),
$$

where $d$ is derivation of $n$-forms. Defining $B:=\bigcup_{k=1}^{\infty} B_{k}$, we have $B=d \Gamma\left(U / R, \Omega_{U / R}^{n}\right)$ filtered by pole order. Note $B_{k} \subseteq B \cap Z_{k}$ but there is not an equality: it fails for singular hypersurfaces; see $\left[7\right.$, Theorem A, (A2)] and the comments which follow. Certainly though, $H_{d R}^{n+1}(U / R)=$ $Z / B$.

Since we are working with the universal hypersurface, the quotient $Z / B$ though presumably finitely generated is complicated. However, by inverting a sequence of polynomials in $R$, one can give an explicit procedure for computing in this quotient based upon reduction of pole order. This is the Griffiths-Dwork method. It has been given a conceptual explanation by Dimca. Filtration by pole order of $\Omega_{U / R}^{\bullet}$ allows one to define a spectral sequence with $E_{\infty}=H_{d R}^{n+1}(U / R)$. This spectral sequence has infinitely many non-zero $E_{1}^{s, t}$ terms for the universal hypersurface, but it degenerates at the $E_{1}$ term $\left(E_{1}=E_{\infty}\right)$ for smooth hypersurfaces (when ' $B_{k}=B \cap Z_{k}$ ') [7, p. 764]. We now give the explicit description.

Let $\Delta_{n, d}^{(n+1)}$ be the 'Macaulay resultant' of the partial derivatives of $P$ defined in $[\mathbf{2 6}, \mathrm{p} .7$, Chapter I.6]. Precisely, let it be Macaulay's determinant ' $D$ ', taking ' $n$ ' to be $n+2$ and ' $F$ ' to be $\delta_{i} P$. Thus, $\Delta_{n, d}^{(n+1)}$ is non-zero but vanishes on the coefficients of homogeneous polynomials of degree $d$ which define singular hypersurfaces of dimension $n$ (and also possibly on the coefficients of some other polynomials). Define $R^{(n+1)}:=R\left[1 / \Delta_{n, d}^{(n+1)}\right]$ and for any $R$-module $\bullet$ let $\bullet(n+1):=\bullet \otimes_{R} R^{(n+1)}$. Then we have

$$
Z_{k}^{(n+1)}=B_{k}^{(n+1)}+Z_{n+1}^{(n+1)} \text { for } k \geqslant n+1 .
$$

This follows since after inverting $\Delta_{n, d}^{(n+1)}$ one may use linear algebra (via the matrix whose determinant is the Macaulay resultant) to reduce any $(n+1)$-form in $Z_{k}^{(n+1)}$ modulo exact 
forms (in $B_{k}^{(n+1)}$ ) to have pole order at most $n+1$. It follows that

$$
Z^{(n+1)} / B^{(n+1)} \cong Z_{n+1}^{(n+1)} /\left(B^{(n+1)} \cap Z_{n+1}^{(n+1)}\right)
$$

and so $Z^{(n+1)} / B^{(n+1)}$ is finitely generated. Note that since certainly $B_{n+1}^{(n+1)} \subseteq B^{(n+1)} \cap Z_{n+1}^{(n+1)}$, we have $Z^{(n+1)} / B^{(n+1)}$ is a quotient of $Z_{n+1}^{(n+1)} / B_{n+1}^{(n+1)}$; in fact, $B_{n+1}^{(n+1)}=B^{(n+1)} \cap Z_{n+1}^{(n+1)}$ by $[7$, Theorem A, (A2)] and the two are equal.

The quotient $Z^{(n+1)} / B^{(n+1)}$ is locally free, but it is not free in general without further localisation which will depend upon a choice of basis. Now $\mathcal{B}_{U}$ is a basis generically since it is a basis for the de Rham cohomology of the complement of the diagonal hypersurface $x_{0}^{d}+\ldots+x_{n+1}^{d}=0$. (Over $\mathbb{C}$, the action of the $(n+2)$-fold product of the group of $d$ th roots of unity decomposes into characters via this basis.) Write $\mathcal{B}_{U, k}:=\mathcal{B}_{U} \cap Z_{k}$, an intersection as sets. One can now construct an explicit Macaulay-style matrix depending on $\mathcal{B}_{U, n+1}$ whose determinant $\Delta_{n, d}^{(n)}$ is such that defining $R^{(n)}:=R^{(n+1)}\left[1 / \Delta_{n, d}^{(n)}\right]$ one has

$$
Z^{(n)} / B^{(n)} \cong Z_{n+1}^{(n)} / B_{n+1}^{(n)} \cong\left\langle\mathcal{B}_{U, n+1}\right\rangle_{R^{(n)}} \oplus\left(Z_{n}^{(n)} / B_{n}^{(n)}\right) .
$$

Here we are following the same convention for base extension of an $R$-module $\bullet$, that is, - $(n):=\bullet \otimes_{R} R^{(n)}$. In words, every $(n+1)$-form with a pole of order at most $n+1$ can be written modulo an exact form of pole order at most $n+1$ as an $R^{(n)}$-linear combination of the basis forms in $\mathcal{B}_{U, n+1}$ plus a form of pole order at most $n$.

This continues inductively, defining $\Delta_{n, d}^{(k)}$ and $R^{(k)}:=R^{(k+1)}\left[1 / \Delta_{n, d}^{(k)}\right]$ for $k=n-1, \ldots, 1$ at each step so that

$$
Z^{(k)} / B^{(k)} \cong\left\langle\bigcup_{\ell=k+1}^{n+1} \mathcal{B}_{U, \ell}\right\rangle_{R^{(k)}} \oplus\left(Z_{k}^{(k)} / B_{k}^{(k)}\right)
$$

and this isomorphism is effectively computable via linear algebra. Finally, noting $Z_{1}^{(1)} / B_{1}^{(1)} \cong$ $\left\langle B_{U, 1}\right\rangle_{R^{(1)}}$, one finds

$$
Z^{(1)} / B^{(1)} \cong\left\langle\mathcal{B}_{U}\right\rangle_{R^{(1)}}
$$

Finally, define $\Delta_{n, d}:=\prod_{k=1}^{n+1} \Delta_{n, d}^{(k)}$, so that $R^{(1)}=R\left[1 / \Delta_{n, d}\right]$, and let $T:=\operatorname{Spec}\left(R^{(1)}\right)$. Writing ' $U / T$ ' to denote $U \otimes_{R} R^{(1)} / R^{(1)}$, one has Theorem 2.6.

Note 2.9. The exact description of the Macaulay-style matrices whose determinants are the polynomials $\Delta_{n, d}^{(k)}$, and the method for using these matrices to make the isomorphisms above explicit, is a little involved; see [22] for the details in a closely analogous situation, or [31] for full details of this construction. The method has been implemented in code by Pancratz and seems well suited to computing Picard-Fuchs systems for families of hypersurfaces; see [31] and the proof of Theorem 2.13. Our computations of Picard-Fuchs systems for the examples in Section 5 actually use a slower implementation due to the author, which exploits the Gröbner basis routines for the rational function field $\mathbb{Q}(t)$ in the programming language MAGMA.

2.3.3. Computation of the Picard-Fuchs system. Let $F$ be a computable field of characteristic zero. Let $P_{t} \in F[t]\left[x_{0}, x_{1}, \ldots, x_{n+1}\right]$ be a homogeneous polynomial of degree $d$ in the variables $x_{0}, x_{1}, \ldots, x_{n+1}$ with coefficients in the polynomial ring $F[t]$. Let $Y_{t} \subset \mathbb{P}_{F(t)}^{n+1}$ be the hypersurface defined by the equation $P_{t}=0$. We shall assume that $Y_{t}$ is smooth and in general position; see Definition 2.7. Denote by $\Delta_{n, d}\left(P_{t}\right) \in F[t]$ the non-zero polynomial obtained by specialising $\Delta_{n, d}$ at the coefficients of $P_{t}$. Let $\Delta(t) \in F[t]$ be any non-zero polynomial which contains $\Delta_{n, d}\left(P_{t}\right)$ as a factor. Define $A:=F[t, 1 / \Delta(t)]$ and $S:=\operatorname{Spec}(A)$. Let $X \subset \mathbb{P}_{S}^{n+1}$ be the scheme defined by $P_{t}=0$ and $U$ be its affine complement. 
Note 2.10. One is really just interested in the case $\Delta(t):=\Delta_{n, d}\left(P_{t}\right)$, but it is harmless to excise some additional fibres and allowing this eases the statement of some results.

Corollary 2.11. The set of cohomology classes $\left\{[b] \mid b \in \mathcal{B}_{U}\right\}$ is an A-basis for $H_{d R}^{n+1}(U / S)$.

Here the $P$ in the definition of $\mathcal{B}_{U}$ should be specialised to $P_{t}$.

Proof. By Theorem 2.6, noting that $H_{d R}^{n+1}(U / R)$ is a quotient $Z / B$ and tensor product of rings $\bullet \otimes_{R^{(1)}} A$ is right exact.

Letting $\mathcal{H}$ denote the primitive part of $H_{d R}^{n}(X / S)$, we deduce the following.

Corollary 2.12. The Dwork basis $\mathcal{B}_{X}$ is an $A$-basis for $\mathcal{H}$.

We can now present our theorem on Picard-Fuchs systems for projective hypersurfaces.

Theorem 2.13. The matrix $N(t)$ for the Gauss-Manin connection $\nabla$ restricted to the primitive middle-dimensional cohomology $\mathcal{H}$ of $X \rightarrow S$ with respect to the Dwork basis $\mathcal{B}_{X}$ is effectively computable.

Proof. Equivalently, we compute the Gauss-Manin connection on the basis of $H_{d R}^{n+1}(U / S)$ given by classes of forms in $\mathcal{B}_{U}$. Partition the basis of differential forms $\mathcal{B}_{U}$ via pole order $k$ along $X$ as before,

$$
\mathcal{B}_{U}=\bigsqcup_{k=1}^{n+1} \mathcal{B}_{U, k} .
$$

For $0 \leqslant k \leqslant n$, let $\mathcal{H}^{k}$ denote the $A$-module spanned by the image of $\bigsqcup_{j=1}^{k+1} \mathcal{B}_{U, j}$ in the primitive middle-dimensional cohomology $\mathcal{H}$ of $X \rightarrow S$. Then $\mathcal{H}^{\bullet}$ is the Hodge filtration on $\mathcal{H}$. The Gauss-Manin connection $\nabla=\nabla_{\frac{d}{d t}}$ is just differentiation of cohomology classes with respect to the parameter followed by reduction in cohomology. Computing $\frac{d}{d t}$ on a basis element is straightforward:

$$
\left[\frac{x^{w} \Omega}{P_{t}^{k}}\right] \stackrel{\frac{d}{d t}}{\longmapsto}\left[\frac{-k x^{w} \frac{d P_{t}}{d t} \Omega}{P_{t}^{k+1}}\right],
$$

where square brackets indicate the cohomology class of a differential form. Reduction in cohomology is performed via linear algebra as in $\S 2.3 .2$, by specialising all of the matrices $\Delta_{n, d}^{(k)}$ at the coefficients in $F[t]$ of the polynomial $P_{t}$.

This explicit description of the computation of the Picard-Fuchs matrix $N(t)$ via linear algebra shows that it has a particular block form. Specifically, for $1 \leqslant k \leqslant n+1$, let $\Delta_{n, d}^{(k)}\left(P_{t}\right)$ denote the specialisation of the polynomial $\Delta_{n, d}^{(k)}$ from $\S 2.3 .2$ at the coefficients of $P_{t}$. Define $\Delta_{n, d}^{(0)}\left(P_{t}\right):=1$.

Corollary 2.14. Let $N(t)$ be the Picard-Fuchs matrix with respect to the Dwork basis, and $N_{k, \ell}(1 \leqslant k, \ell \leqslant n+1)$ the block of entries which gives for $b \in \mathcal{B}_{U, \ell}$ the coefficients in $\nabla(b)$ of the elements in $\mathcal{B}_{U, k}$. Then for $k-1 \leqslant \ell$ the matrix

$$
\left(\prod_{j=k-1}^{\ell} \Delta_{n, d}^{(j)}\left(P_{t}\right)\right) N_{k, \ell}
$$

has entries in $F[t]$. For $k>\ell+1$, we have $N_{k, \ell}=0$ (Griffiths's transversality). 
Proof. Let $b \in \mathcal{B}_{U, \ell}$. Then, by $(2.2), \nabla(b)$ can be represented by a differential form with pole order $\ell+1$ along $X$. Now use the explicit pole reduction method at the end of $\S 2.3 .2$, specialising $P$ to $P_{t}$ and tensoring $R$-modules by $\bullet \otimes_{R} F[t]$, to write this as an $F(t)$-linear combination of the basis $\mathcal{B}_{U}$, dividing by $\Delta_{n, d}^{(j)}\left(P_{t}\right)(1 \leqslant j \leqslant \ell)$ when reducing pole orders from $j+1$ to $j$ modulo exact forms. Precisely, when one has reduced modulo exact forms to a get pole of order $k$ plus an $F\left[t, 1 / \prod_{j=k}^{\ell} \Delta_{n, d}^{(j)}\left(P_{t}\right)\right]$-linear combination of $\bigcup_{j=k+1}^{\ell} \mathcal{B}_{U, j}$, one needs to divide by $\Delta_{n, d}^{(k-1)}\left(P_{t}\right)$ to obtain an $F\left[t, 1 / \prod_{j=k-1}^{\ell} \Delta_{n, d}^{(j)}\left(P_{t}\right)\right]$-linear combination of $\bigcup_{j=k}^{\ell} \mathcal{B}_{U, j}$ modulo an exact form and a form of pole order $k-1$, cf. (2.1).

In particular, provided that $\Delta_{n, d}\left(P_{t}\right)$ is squarefree, then the Picard-Fuchs matrix has simple poles at the finite points. This is true generically if $\Delta_{n, d}$ itself is squarefree, which in turn follows if one finds one specialisation of maximum degree which is squarefree; that is, one suitable family. The author expects that a Lefschetz pencil would work, but he has not proved this.

2.3.4. Semistable reduction for hypersurfaces. We come to the main theorem of $\S 2.3$. Recall that for $e$ a positive integer and $S=\operatorname{Spec}(F[t, 1 / \Delta(t)])$, we define $S_{e}:=\operatorname{Spec}\left(F\left[s, 1 / \Delta\left(s^{e}\right)\right]\right)$ with $S_{e} \rightarrow S$ defined by $t \mapsto s^{e}$ and write $X_{e}:=X \times{ }_{S} S_{e}$.

TheOREm 2.15. There exists an explicit deterministic algorithm with the following input and output. The input is a homogeneous polynomial $P_{t} \in F[t]\left[x_{0}, x_{1}, \ldots, x_{n}\right]$ and a polynomial $\Delta(t) \in F[t]$ such that writing $S:=\operatorname{Spec}(F[t, 1 / \Delta(t)])$ the fibres in the family $X \rightarrow S$ defined by $P_{t}$ are smooth and in general position. The output is a positive integer $e$, a basis $\mathcal{B}_{e}$ for the primitive middle-dimensional cohomology of $X_{e} \rightarrow S_{e}$ and a matrix $N(t)$ for the GaussManin connection $\nabla_{e}$ on this basis which has only a simple pole at $s=0$ with nilpotent residue matrix.

Proof. By Theorem 2.13, one can compute the matrix for the Gauss-Manin connection $\nabla$ on the primitive middle-dimensional cohomology of $X \rightarrow S$. If $\Delta(0) \neq 0$, then we are done. Otherwise, Theorem 2.4 performs the necessary pull back $S_{e} \rightarrow S$ and change of basis defined over the whole of $S_{e}$.

\section{Degenerations in positive characteristic}

Let $\mathbb{F}_{q}$ be the finite field with $q$ elements of characteristic $p, K$ be the unique unramified

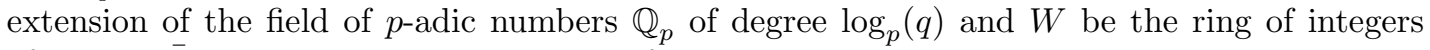
of $K$. Let $\bar{K}$ denote an algebraic closure of $K$.

\subsection{Relative rigid cohomology}

Let $\mathcal{X} \rightarrow \mathcal{S}$ be a morphism of $W$-schemes, where $\mathcal{S}$ is an open subscheme of $\mathbb{A}_{W}^{1}$.

Definition 3.1. We shall say that 'Assumption (SP) holds' if either $\mathcal{X} \rightarrow \mathcal{S}$ is smooth and proper, or it is the restriction of a smooth and proper morphism to the complement of a normal crossings divisor with smooth components over $\mathcal{S}$.

We shall assume throughout Section 3 that Assumption (SP) holds. Write $\mathcal{S}:=\operatorname{Spec}(A)$, where $A=W[t, 1 / \Delta(t)]$ for some polynomial $\Delta(t) \in W[t]$. We assume that $p$ does not divide $\Delta(t)$. Let $\mathcal{X}_{K} \rightarrow \mathcal{S}_{K}$ and $X \rightarrow S$ denote maps on generic and special fibres, respectively. Let $A_{K}:=A \otimes_{W} K$ and define $A_{K}^{\dagger}$ to be the weak completion of $A$ tensored by $K$. Precisely, denote the completion of $A$ with respect to the Gaussian norm $\left[\mathbf{9}\right.$, p. 10] by $\hat{A}$. Then $A_{K}^{\dagger}$ consists of 
series in $\hat{A} \otimes_{W} K$ which converge on $\mathbb{P}_{\bar{K}}^{1}$ with open disks of some unspecified radius less than one around the roots of $\Delta(t)$ and infinity removed. Thus, $A_{K}^{\dagger}$ has a $p$-adic valuation $\operatorname{ord}_{p}(\cdot)$, and one may extend this to matrix rings over $A_{K}^{\dagger}$ in the obvious way. Define the Frobenius $\sigma: A_{K}^{\dagger} \rightarrow A_{K}^{\dagger}$ by $\sigma: t \mapsto t^{p}$ and let it act on coefficients by the usual Frobenius automorphism of $K$.

Let $H_{\mathrm{rig}}^{m}(X / S)$ denote the $m$ th relative rigid cohomology of $X \rightarrow S$. (We refer to $[\mathbf{1 0}$, Section 2], [23, Section 3] and [24] for details and references on the construction of relative rigid cohomology.) This is a locally free $A_{K}^{\dagger}$-module of finite rank equipped with a connection $\nabla_{\text {rig }}$ and $\sigma$-linear Frobenius $\mathcal{F}$ which commute, in the following sense. By Assumption (SP), we have the comparison theorem [23, Theorem 3.1]

$$
H_{\text {rig }}^{m}(X / S) \cong H_{d R}^{m}\left(\mathcal{X}_{K} / \mathcal{S}_{K}\right) \otimes_{A_{K}} A_{K}^{\dagger} .
$$

Since $H_{d R}^{m}\left(\mathcal{X}_{K} / \mathcal{S}_{K}\right)$ is a locally free module over a principal ideal domain, it is free. Thus, $H_{\text {rig }}^{m}(X / S)$ is actually a free $A_{K}^{\dagger}$-module. Let $\mathcal{B}$ be a basis for $H_{d R}^{m}\left(\mathcal{X}_{K} / \mathcal{S}_{K}\right)$ and $N(t)$ the matrix for the Gauss-Manin connection $\nabla$ with respect to $\mathcal{B}$. Write $\mathcal{B}_{\text {rig }}:=\left\{b \otimes 1_{A_{K}^{\dagger}}\right\}$ for the associated basis of $H_{\text {rig }}^{m}(X / S)$. Then by the comparison theorem the matrix for $\nabla_{\text {rig }}$ with respect to $\mathcal{B}_{\text {rig }}$ is also $N(t)$. Let $F(t)$ denote the matrix for the Frobenius $\mathcal{F}$ with respect to $\mathcal{B}_{\text {rig. }}$. Thus, $F(t)$ has entries in $A_{K}^{\dagger}$. The matrix $F(t)$ is invertible and

$$
\frac{d F}{d t}+N(t) F(t)=F(t) \sigma(N(t)) p t^{p-1} .
$$

This equation exactly describes the 'commutativity' of $\nabla_{\text {rig }}$ and $\mathcal{F}$.

Denote by $\mathcal{R}$ the Robba ring around $t=0$ [17, Chapter 15.1]. That is, the ring of two-way infinite Laurent series with coefficients in $K$ which converge on some open annulus of outer radius one and unspecified inner radius $r<1$ around the origin. From the definition of $A_{K}^{\dagger}$, one sees that there is an embedding $A_{K}^{\dagger} \hookrightarrow \mathcal{R}$ via local expansions. Letting $K((t))$ denote the usual ring of one-way infinite Laurent series, one may embed $A_{K} \hookrightarrow K((t))$. Thus, the entries of $N(t)$ and $F(t)$ may be expanded locally around $t=0$ to give elements in $K((t))$ and $\mathcal{R}$, respectively.

We note that relative rigid cohomology here depends only upon the morphism $X \rightarrow S$ and not upon the lift $\mathcal{X} \rightarrow \mathcal{S}$. This follows since there is a specialisation theorem (see [23, Theorem 3.2] or [15, Theorem 6.1.3]) comparing relative rigid cohomology with the rigid cohomology along the fibres, and the latter is independent of the lift chosen. Thus, our limiting Frobenius structure defined in the next section actually depends just upon the morphism $X \rightarrow S$ and not upon the choice of lift $\mathcal{X} \rightarrow \mathcal{S}$. It is an interesting question, raised by the referee, as to whether relative rigid cohomology [24, Chapter 8] in general is independent of all auxiliary choices made in its construction. One approach to answering this affirmatively is through Le Stum's construction of the overconvergent site, in particular [25, Proposition 3.5.2, Theorem 3.5.3]; however, this is not directly relevant to our work and beyond the author's expertise.

\subsection{Limiting Frobenius structures}

3.2.1. A surprising lemma. Suppose $\Delta(0)=0$ : then when one embeds entries in the matrix $F(t)$ into the Robba ring $\mathcal{R}$ one expects infinitely many non-zero negative power terms in their Laurent series expansions. The following result is surprising and rather deep.

Lemma 3.2. Let $N(t)=\sum_{i=-1}^{\infty} N_{i} t^{i}$ be an $r \times r$ matrix such that the entries of $t N$ are power series over $K$ convergent in the open unit disk, and $N_{-1}$ is a nilpotent matrix. Let $F(t)=\sum_{i=-\infty}^{\infty} F_{i} t^{i}$ be an $r \times r$ matrix whose entries are (two-way infinite) Laurent series over $K$ convergent on some open annulus with outer radius 1 . Suppose that $N$ and $F$ satisfy (3.2). Then $F_{i}=0$ for $i<0$, so $F(t)$ converges on the whole open unit disk. 
Proof. This is exactly [15, Lemma 6.5.7], which in turn is a consequence of $[\mathbf{1 7}$, Proposition 17.5.1]. The proof has two steps. First, one assumes $t N(t)=\mathcal{N}_{0}$, a nilpotent matrix. Then (3.2) becomes

$$
t \frac{d F}{d t}+\mathcal{N}_{0} F=p F \sigma\left(\mathcal{N}_{0}\right)
$$

Now compare coefficients of $t^{i}$ on both sides and observe that the map on matrices $X \mapsto$ $\mathcal{N}_{0} X+X\left(i-p \sigma\left(\mathcal{N}_{0}\right)\right)$ has all eigenvalues equal to $i$ since $\mathcal{N}_{0}$ and $\sigma\left(\mathcal{N}_{0}\right)$ are nilpotent, by $[\mathbf{9}$, Lemma III.8.4]. Hence, we see that $F_{i}=0$ for $i \neq 0$. Next, to reduce the general case to the case $t N(t)=\mathcal{N}_{0}$, one uses a deep 'transfer theorem' of Christol and Dwork [17, Theorem 13.7.1].

Note 3.3. The choice of Frobenius lift $t \mapsto t^{p}$ is key to the proof - this was stressed to the author by Kiran Kedlaya. In the original deformation algorithm, the author used the lift $s \mapsto s^{p}$, where $s$ is the local parameter around $t=1$; that is, $t \mapsto(t-1)^{p}+1$. With this choice of lift, one expects an essential singularity at $t=0$ in $F(t)$ unless the family $X / S$ has a "smooth extension' over the origin. This simple observation is the key to extending the deformation method to the case of degenerations - it eluded the author for many years.

3.2.2. Definition and uniqueness. We now define our limiting Frobenius structures. Note that in this section all bases $\mathcal{B}_{\text {rig }}$ for $H_{\text {rig }}^{m}(X / S)$ arise by extension of scalars from a basis $\mathcal{B}$ of $H_{d R}^{m}\left(\mathcal{X}_{K} / \mathcal{S}_{K}\right)$.

Definition 3.4. Let $F(t)$ and $N(t)$ be the matrices for the Frobenius and connection, respectively, with respect to some basis $\mathcal{B}_{\text {rig }}$ of $H_{\text {rig }}^{m}(X / S)$. Assume that the hypotheses of Lemma 3.2 are satisfied. Write $\mathcal{N}_{0}:=(t N(t))_{t=0}$ for the nilpotent residue matrix, $\mathcal{F}_{0}:=F(0)$ and $\mathcal{H}_{0}:=\left\langle\mathcal{B}_{\text {rig }}\right\rangle_{K}$. Then by $(3.2)$ we have $\mathcal{N}_{0} \mathcal{F}_{0}=p \mathcal{F}_{0} \sigma\left(\mathcal{N}_{0}\right)$. We call $\left(\mathcal{H}_{0}, \mathcal{N}_{0}, \mathcal{F}_{0}\right)$ the limiting Frobenius structure (in dimension $m$ of the family $\mathcal{X} \rightarrow \mathcal{S}$ ) at $t=0$ with respect to the basis $\mathcal{B}_{\text {rig. }}$.

Lemma 3.5. With notation and hypotheses as in Definition 3.4, the matrix $\mathcal{F}_{0}$ is invertible.

Proof. Define $M:=-N^{t}$ and $G:=\left(F^{-1}\right)^{t}$. Then the pair $(M, G)$ is the 'dual overconvergent $F$-isocrystal' and satisfies the differential equation

$$
\frac{d G}{d t}+M(t) G(t)=G(t) \sigma(M(t)) p t^{p-1} .
$$

Now $t M(t)$ converges on the $p$-adic open unit disk and $M(t)$ has residue matrix $-\mathcal{N}_{0}^{t}$, which is nilpotent. Hence, by Lemma 3.2, $G(t)=\sum_{i=0}^{\infty} G_{i} t^{i}$ converges on the open unit disk. But $F G^{t}=I$ and so $F_{0} G_{0}^{t}=I$ and $\mathcal{F}_{0}$ is invertible.

Lemma 3.6. Assume that there are two bases $\mathcal{B}_{\text {rig }}$ and $\mathcal{B}_{\text {rig }}^{\prime}$ which satisfy the hypothesis in Definition 3.4. Let $\left(\mathcal{H}_{0}, \mathcal{N}_{0}, \mathcal{F}_{0}\right)$ and $\left(\mathcal{H}_{0}^{\prime}, \mathcal{N}_{0}^{\prime}, \mathcal{F}_{0}^{\prime}\right)$ be the associated limiting Frobenius structures at $t=0$. Then there exists an invertible matrix $H_{0}$ over $K$ such that $\mathcal{N}_{0}^{\prime}=H_{0} \mathcal{N}_{0} H_{0}^{-1}$ and $\mathcal{F}_{0}^{\prime}=H_{0} \mathcal{F}_{0} \sigma\left(H_{0}\right)$.

Proof. Let $H(t)$ be the local expansion around the origin of the change of basis matrix from $\mathcal{B}$ to $\mathcal{B}^{\prime}$; hence also from $\mathcal{B}_{\text {rig }}$ to $\mathcal{B}_{\text {rig }}^{\prime}$. Let $N(t)$ and $N(t)^{\prime}$ denote the connection matrices with respect to the two bases, and $\mathcal{N}_{0}=(t N(t))_{t=0}$ and $\mathcal{N}_{0}^{\prime}=\left(t N(t)^{\prime}\right)_{t=0}$ the two nilpotent matrices. (Beware here and elsewhere that the prime superscript has nothing to do with differentiation.) Then $\left(t N^{\prime}\right)=H(t N) H^{-1}+t \frac{d H}{d t} H^{-1}$ and so $\left(t N^{\prime}\right) H-H(t N)=t \frac{d H}{d t}$. Write $H(t)=H_{-s} t^{-s}+$ $\ldots$ as a Laurent series with matrix coefficients, where $H_{-s} \neq 0$. So, $\mathcal{N}_{0}^{\prime} H_{-s}-H_{-s} \mathcal{N}_{0}=-s H_{-s}$. But the operator on matrices $X \mapsto \mathcal{N}_{0}^{\prime} X-X \mathcal{N}_{0}$ has eigenvalues the difference of the eigenvalues of the two nilpotent matrices $\mathcal{N}_{0}$ and $\mathcal{N}_{0}^{\prime}$, by [9, Lemma III.8.4]. That is, its eigenvalues are 
all zero. So, $s=0$ and $H$ has entries in $K[[t]]$. By a symmetrical argument, so does $H^{-1}$ and so $H(t)=H_{0}+H_{1} t+\ldots$, where $H_{0}$ is invertible. Thus, $\mathcal{N}_{0}^{\prime}=H_{0} \mathcal{N}_{0} H_{0}^{-1}$. Since the Frobenius map $\mathcal{F}$ is $\sigma$-linear, the change of basis formula shows $\mathcal{F}_{0}^{\prime}=H_{0} \mathcal{F}_{0} \sigma\left(H_{0}\right)$.

Thus, we may speak of 'the' limiting Frobenius structure $\left(\mathcal{H}_{0}, \mathcal{N}_{0}, \mathcal{F}_{0}\right)$ as an abstract triple of a vector space $\mathcal{H}_{0}$ over $K$, a nilpotent linear operator $\mathcal{N}_{0}$ and an invertible $\sigma$-linear operator $\mathcal{F}_{0}$ such that $\mathcal{N}_{0} \mathcal{F}_{0}=p \mathcal{F}_{0} \mathcal{N}_{0}$, provided that it exists with respect to at least one basis.

3.2.3. Existence. The limiting Frobenius structure might only exist after making a cover of the base. We now prove the existence of a 'smallest' limiting Frobenius structure under a lifting hypothesis.

For $e$ a positive integer, write $A_{e}:=W\left[s, 1 / \Delta\left(s^{e}\right)\right],\left(A_{K}\right)_{e}:=K\left[s, 1 / \Delta\left(s^{e}\right)\right]$ and $\left(A_{K}^{\dagger}\right)_{e}$ for the weak completion of $A_{e}$ tensored by $K$. Define $A \rightarrow A_{e}, A_{K} \rightarrow\left(A_{K}\right)_{e}$ and $A_{K}^{\dagger} \rightarrow\left(A_{K}^{\dagger}\right)_{e}$ by $t \mapsto s^{e}$. Let $\mathcal{S}_{e}:=\operatorname{Spec}\left(A_{e}\right)$, so there is a morphism $\mathcal{S}_{e} \rightarrow \mathcal{S}$. One may pull back the family $\mathcal{X} \rightarrow \mathcal{S}$ via $\mathcal{S}_{e} \rightarrow \mathcal{S}$ to get $\mathcal{X}_{e} \rightarrow \mathcal{S}_{e}$, where $\mathcal{X}_{e}:=\mathcal{X} \times \mathcal{S}_{\mathcal{S}} \mathcal{S}_{e}$. Write $X_{e} \rightarrow S_{e}$ and $\left(\mathcal{X}_{K}\right)_{e} \rightarrow\left(\mathcal{S}_{K}\right)_{e}$ for the maps on special and generic fibres. The morphism $\mathcal{X}_{e} \rightarrow \mathcal{S}_{e}$ is smooth (even when $p$ divides $e$ and $\mathcal{S}_{e} \rightarrow \mathcal{S}$ is not) and we have as before

$$
H_{\text {rig }}^{m}\left(X_{e} / S_{e}\right) \cong H_{d R}^{m}\left(\left(\mathcal{X}_{K}\right)_{e} /\left(\mathcal{S}_{K}\right)_{e}\right) \otimes_{\left(A_{K}\right)_{e}}\left(A_{K}^{\dagger}\right)_{e} .
$$

Definition 3.7. Assume that the hypotheses of Definition 3.4 are satisfied for the family $\mathcal{X}_{e} \rightarrow \mathcal{S}_{e}$ after pulling back the base $\mathcal{S}_{e} \rightarrow \mathcal{S}$ for some positive integer $e$. Let $\left(\mathcal{H}_{0}, \mathcal{N}_{0}, \mathcal{F}_{0}\right)$ be the limiting Frobenius structure for $\mathcal{X}_{e} \rightarrow \mathcal{S}_{e}$ at $s=0$. We call $\left(\mathcal{H}_{0}, \mathcal{N}_{0}, \mathcal{F}_{0}, e\right)$ a limiting Frobenius structure for $\mathcal{X} \rightarrow \mathcal{S}$ at $t=0$.

Note that if one has a chain of covers $\mathcal{S}_{e^{\prime}} \rightarrow \mathcal{S}_{e} \rightarrow \mathcal{S}$ and a limiting Frobenius structure $\left(\mathcal{H}_{0}, \mathcal{N}_{0}, \mathcal{F}_{0}, e\right)$ 'on' $\mathcal{S}_{e}$, one may pull it back to a limiting Frobenius structure $\left(\mathcal{H}_{0},\left(e^{\prime} / e\right) \mathcal{N}_{0}, \mathcal{F}_{0}, e^{\prime}\right)$ 'on' $\mathcal{S}_{e^{\prime}}$. We then say that the latter factors through the former.

We now state our main theorem on the existence of limiting Frobenius structures. Recall that Assumption (SP) is still in place. We shall call the $p$-adic open unit disk around $t=0$ with the origin removed the 'punctured' $p$-adic open unit disk around $t=0$.

TheOREm 3.8. Assume that the polynomial $\Delta(t)$ has no zeros in $\bar{K}$ within the punctured $p$-adic open unit disk around $t=0$. Then a limiting Frobenius structure $\left(\mathcal{H}_{0}, \mathcal{N}_{0}, \mathcal{F}_{0}, e\right)$ at $t=0$ exists. Moreover, there exists a unique 'smallest' such limiting Frobenius structure, such that any other factors through it.

Proof. Let $\mathcal{B}$ be any basis for $H_{d R}^{m}\left(\mathcal{X}_{K} / \mathcal{S}_{K}\right), r$ the size of this basis and $N(t)$ the matrix for the Gauss-Manin connection with respect to this basis. The matrix $N(t)$ converges on the punctured $p$-adic open unit disk around $t=0$, since it has poles only at the roots of $\Delta(t)$. Theorem 2.4 guarantees the existence of a smallest positive integer $e$ and change of basis matrix $H \in \mathrm{GL}_{r}\left(K\left[s, s^{-1}\right]\right.$ ) (here $\left.t=s^{e}\right)$ such that the matrix for the Gauss-Manin connection on $\left(\mathcal{B}_{e}\right)_{[H(s)]}$ has only a simple pole at $s=0$ with nilpotent residue matrix. Here $e$ is taken to be the lowest common multiple of the denominators of the eigenvalues $\lambda_{i}$ of the residue matrix which occur in the proof of Theorem 2.4. (Note that the hypothesis of computability on the base field $F$ is only used to establish that the change of basis matrix $H(s)$ is computable.) Denote this connection matrix by $N(s)^{\prime}$. Since $H \in \mathrm{GL}_{r}\left(K\left[s, s^{-1}\right]\right)$, the matrix $N(s)^{\prime}$ converges on the punctured $p$-adic open unit disk around $s=0$. Thus, the hypotheses of Definition 3.7 apply after making the base change $\mathcal{S}_{e} \rightarrow \mathcal{S}$ and we may define a limiting Frobenius structure at $t=0$. 
A necessary condition for the existence of a limiting Frobenius structure after pulling back the base $\mathcal{S}_{e^{\prime}} \rightarrow \mathcal{S}$ is that $e^{\prime} \lambda_{i}$ are integers. Hence, for any such $e^{\prime}$, we have that $e$ divides $e^{\prime}$; hence, $\mathcal{S}_{e^{\prime}} \rightarrow \mathcal{S}$ factors through $\mathcal{S}_{e} \rightarrow \mathcal{S}$. Thus, we can pull back the limiting Frobenius structure $\left(\mathcal{H}_{0}, \mathcal{N}_{0}, \mathcal{F}_{0}, e\right)$ via $\mathcal{S}_{e^{\prime}} \rightarrow \mathcal{S}_{e}$ and apply Lemma 3.6 for the final claim.

We call the 'smallest' limiting Frobenius structure which occurs in Theorem 3.8, 'the' limiting Frobenius structure at $t=0$.

Note 3.9. Provided that the characteristic $p$ does not divide $e$, the morphism $\mathcal{S}_{e} \rightarrow \mathcal{S}$ reduces to an unramified cover $S_{e} \rightarrow S$. Thus, here we have made the overconvergent $F$-isocrystal $H_{\text {rig }}^{m}(X / S)$ 'unipotent around zero' after making an unramified cover of the base $S$. So, our construction here agrees with one arising from the semistable reduction theorem. If $p$ divides $e$, then our construction seems unnatural, since the cover $S_{e} \rightarrow S$ is totally ramified. The author suspected that the hypothesis on $\Delta(t)$ in Theorem 3.8 could not be satisfied when the order $e$ of the finite part of the local monodromy around the origin of the family $\mathcal{X}_{K} \rightarrow \mathcal{S}_{K}$ was divisible by $p$. The referee and Bruno Chiarellotto explained to him why this was so: since one has a Frobenius structure in which the connection has no singularity in an open unit disk except at the central point, then by Théorème 5.5-3 and the first part of $[4, \mathrm{p} .404]$ the eigenvalues of the residue matrix (exponents) are $p$-adically integral.

Let us conclude with a definition which brings together the properties required for Theorem 3.8 to hold.

Definition 3.10. We shall say that a morphism $X \rightarrow S \subseteq \mathbb{A}_{\mathbb{F}_{q}}^{1}$ of $\mathbb{F}_{q}$-varieties has a good lift around zero if it arises by base change from a morphism $\mathcal{X} \rightarrow \mathcal{S}$ of $W$-schemes with the following property. Assumption (SP) is satisfied (Definition 3.1) and, writing $\mathcal{S}=\operatorname{Spec}(W[t, 1 / \Delta(t)]$ ), the polynomial $\Delta(t)$ has no zeros in $\bar{K}$ within the punctured $p$-adic open unit disk around zero.

So, by Theorem 3.8, limiting Frobenius structures exist when one has a good lift around zero.

3.2.4. Computability: definitions and a sketch. We now turn to the question of computing limiting Frobenius structures. The field $K$ is uncountable and thus we immediately encounter two problems. First, one cannot perform exact arithmetic in $K$. Second, we cannot directly apply the results of Section 2, since there the base field $F$ is assumed to be countable. A convenient solution to both problems is to work in a suitable algebraic number field $L \subset K$, using exact arithmetic in $L$ when we wish to apply the 'algebraic' theory from Section 2, and approximating elements in $K$ by those in $L$ during the 'analytic' parts of the algorithm.

Precisely, assume that we have an algebraic number field $L$ with ring of integers $\mathcal{O}_{L}$ in which the prime $p$ is inert with residue field $\mathbb{F}_{q}$. We have the natural embedding $\mathcal{O}_{L} \hookrightarrow W$ given by localisation at $p$. First, we give an 'algebraic' version of Definition 3.10.

Definition 3.11. We shall say that a morphism $X \rightarrow S \subseteq \mathbb{A}_{\mathbb{F}_{q}}^{1}$ of $\mathbb{F}_{q^{-} \text {-varieties has a good }}$ algebraic lift around zero if it arises by base change from a morphism $\mathcal{X}_{\mathcal{O}_{L}} \rightarrow \mathcal{S}_{\mathcal{O}_{L}}$ of $\mathcal{O}_{L^{-}}$ schemes with the following property. Assumption (SP) is satisfied for $\mathcal{X}:=\mathcal{X}_{\mathcal{O}_{L}} \times_{\mathcal{O}_{L}} W \rightarrow \mathcal{S}:=$ $\mathcal{S}_{\mathcal{O}_{L}} \times_{\mathcal{O}_{L}} W$ (Definition 3.1) and, writing $\mathcal{S}_{\mathcal{O}_{L}}=\operatorname{Spec}\left(\mathcal{O}_{L}[t, 1 / \Delta(t)]\right)$, the polynomial $\Delta(t)$ has no zeros in $\bar{K}$ within the punctured $p$-adic open unit disk around zero.

When we have a good algebraic lift $\mathcal{X}_{\mathcal{O}_{L}} \rightarrow \mathcal{S}_{\mathcal{O}_{L}}$, write $\mathcal{X}_{L} \rightarrow \mathcal{S}_{L}$ for the base change by - $\otimes_{\mathcal{O}_{L}} L$. By Theorem 3.8, 'the' limiting Frobenius structure $\left(\mathcal{H}_{0}, \mathcal{N}_{0}, \mathcal{F}_{0}, e\right)$ exists for $\mathcal{X} \rightarrow \mathcal{S}$ when one has a good algebraic lift around zero. Unfortunately, we cannot hope to compute it, 
since any matrix for $\mathcal{F}_{0}$ has entries in the uncountable field $K$. Thus, we need a notion of an 'approximate' limiting Frobenius structure.

For $N$ and $r$ positive integers and $\mathcal{F}_{0} \in \mathrm{GL}_{r}(K)$, we shall call a matrix $\tilde{\mathcal{F}}_{0} \in \mathrm{GL}_{r}(L)$ a $p^{N}$-approximation to $\mathcal{F}_{0}$ if $\operatorname{ord}_{p}\left(\tilde{\mathcal{F}}_{0}-\mathcal{F}_{0}\right) \geqslant N$.

Definition 3.12. Let $N$ be a positive integer. Assume that $X \rightarrow S$ has a good algebraic lift around zero. A $p^{N}$-approximation to the limiting Frobenius structure at $t=0$ in dimension $m$ for the smooth morphism $\mathcal{X} \rightarrow \mathcal{S}$ is a quadruple $\left(\mathcal{H}_{0}, \mathcal{N}_{0}, \tilde{\mathcal{F}}_{0}, e\right)$ with the following properties:

- $\mathcal{H}_{0}$ is a vector space over $K, \mathcal{N}_{0}$ a nilpotent matrix over $L$ and $\tilde{\mathcal{F}}_{0}$ an invertible matrix over $L$;

- the limiting Frobenius structure for $\mathcal{X} \rightarrow \mathcal{S}$ in dimension $m$ at $t=0$ exists and, with respect to some basis, is $\left(\mathcal{H}_{0}, \mathcal{N}_{0}, \mathcal{F}_{0}, e\right)$ with $\tilde{\mathcal{F}}_{0}$ a $p^{N}$-approximation to $\mathcal{F}_{0}$.

The 'algebraic' part of the algorithm for computing approximate limiting Frobenius structures is to find an integer $e$ and a basis for $H_{d R}^{m}\left(\left(\mathcal{X}_{L}\right)_{e} /\left(\mathcal{S}_{L}\right)_{e}\right)$ such that the matrix for the connection has a simple pole at $s=0$ with nilpotent residue matrix. (We use analogous notation for pulling back $\mathcal{S}_{L}$-schemes via the morphism $t \mapsto s^{e}$ as before; that is, introduce a subscript $e$.) This allows us to compute exactly the nilpotent monodromy matrix $\mathcal{N}_{0}$, and of course the integer $e$. The following theorem shows that this can be accomplished provided that we know the connection matrix on some initial basis for $H_{d R}^{m}\left(\mathcal{X}_{L} / \mathcal{S}_{L}\right)$.

Theorem 3.13. There exists an explicit deterministic algorithm with the following input and output. The input is a matrix for the Gauss-Manin connection $\nabla$ with respect to some basis of $H_{d R}^{m}\left(\mathcal{X}_{L} / \mathcal{S}_{L}\right)$, where $X \rightarrow S$ has a good algebraic lift around $t=0$. The output is a matrix $N(s)$ for $\nabla_{e}$ with respect to some explicit basis of $H_{d R}^{m}\left(\left(\mathcal{X}_{L}\right)_{e} /\left(\mathcal{S}_{L}\right)_{e}\right)$ such that $N(s)$ converges on the punctured $p$-adic open unit disk around $s=0$ and has a simple pole at $s=0$ with nilpotent residue matrix.

Proof. Let $\mathcal{B}$ be the basis used for the input. Then the connection matrix with respect to this basis has only poles at the roots of $\Delta(t)$ and thus converges on the punctured $p$-adic open unit disk around $t=0$. Now we apply Theorem 2.4 exactly as in the proof of Theorem 3.8, noting that $L$ is computable.

The 'analytic' part of the algorithm is to compute a suitable approximation to the matrix for Frobenius on this basis. Let us first formalise our notion of approximate Frobenius matrices. For $e \geqslant 1$, define as before $\left(A_{K}^{\dagger}\right)_{e}$ to be the weak completion of $W\left[s, 1 / \Delta\left(s^{e}\right)\right]$ tensored by $K$. Write $\left(A_{L}\right)_{e}:=L\left[s, 1 / \Delta\left(s^{e}\right)\right]$ and note $\left(A_{L}\right)_{e} \hookrightarrow\left(A_{K}^{\dagger}\right)_{e}$. Let $N$ and $r$ be positive integers. We call $\tilde{F}(s) \in \mathrm{GL}_{r}\left(\left(A_{L}\right)_{e}\right)$ a $p^{N}$-approximation to $F(s) \in \mathrm{GL}_{r}\left(\left(A_{K}^{\dagger}\right)_{e}\right)$ if $\operatorname{ord}_{p}(\tilde{F}(s)-F(s)) \geqslant N$. Thus, our aim is to compute a $p^{N}$-approximation $\tilde{F}(s)$ to the matrix $F(s)$ for Frobenius on this basis, and then 'specialise' this approximation at $s=0$ to obtain a $p^{N}$-approximation $\tilde{\mathcal{F}}_{0}$ to $\mathcal{F}_{0}$. The approximation $\tilde{F}(s)$ can be found using the original deformation method.

Rather than state a formal theorem on approximate computability of limiting Frobenius structures in this general setting, let us focus now on the case of degenerations of projective hypersurfaces.

\subsection{Hypersurfaces}

In this section, we prove our main result (Theorem 3.15) on the computability of limiting Frobenius structures for degenerations of hypersurfaces, and discuss the asymptotic complexity of the algorithm underlying this theorem (§3.3.2). 
3.3.1. Computability of limiting Frobenius structures. Let us make a convenient definition to ease the statement of our main theorem. As before, $\mathcal{O}_{L}$ is the ring of integers of an algebraic number field $L$ which is inert at the rational prime $p$ with residue field $\mathbb{F}_{q}$, and $\mathcal{O}_{L} \hookrightarrow W$ is the embedding. Now take $F$ to be the computable field $L$ and define notation in a similar manner to the first paragraph of $\S 2.3 .3$ but taking $P_{t}$ to have coefficients in $\mathcal{O}_{L}[t]$ instead of $L[t]$. Precisely, let $\Delta(t) \in \mathcal{O}_{L}[t]$ be any polynomial which has as a factor the polynomial $\Delta_{n, d}\left(P_{t}\right)$ defined by specialising $\Delta_{n, d}$ at the coefficients of $P_{t}$. Assume $\Delta(t) \neq 0$. Let $\mathcal{S}_{\mathcal{O}_{L}}:=\operatorname{Spec}\left(\mathcal{O}_{L}[t, 1 / \Delta(t)]\right)$

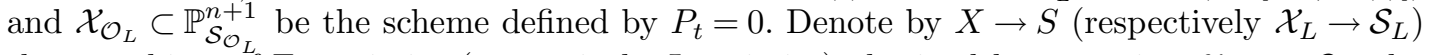
the morphism of $\mathbb{F}_{q}$-varieties (respectively $L$-varieties) obtained by tensoring $\mathcal{X}_{\mathcal{O}_{L}} \rightarrow \mathcal{S}_{\mathcal{O}_{L}}$ by - $\otimes_{\mathcal{O}_{L}} \mathbb{F}_{q}$ (respectively $\left.\bullet \otimes_{\mathcal{O}_{L}} L\right)$. So, each fibre in the family $\mathcal{X}_{L} \rightarrow \mathcal{S}_{L}$ is smooth and in general position (Definition 2.7). Now make the additional assumptions that $\Delta(1) \neq 0 \bmod p$ and $\Delta(t)$ has no zeros in the punctured $p$-adic open unit disk around $t=0$.

Definition 3.14. We shall say that a smooth family of hypersurfaces $X \rightarrow S$ of degree $d$ over $\mathbb{F}_{q}$ is in general position and has a good algebraic lift around $t=0$ if it arises by reduction modulo $p$ from a morphism $\mathcal{X}_{\mathcal{O}_{L}} \rightarrow \mathcal{S}_{\mathcal{O}_{L}}$ of $\mathcal{O}_{L}$-schemes of the type described in the paragraph immediately above.

TheOREM 3.15. There exists an explicit deterministic algorithm which takes as input a positive integer $N$ and a smooth family of hypersurfaces $X \rightarrow S$ over $\mathbb{F}_{q}$ which is in general position and has a good algebraic lift around $t=0$, and gives as output a $p^{N}$-approximation to the primitive middle-dimensional limiting Frobenius structure at $t=0$ of the family $X \rightarrow S$.

Proof. Let $\mathcal{B}:=\mathcal{B}_{\mathcal{X}_{L}}$ be the Dwork basis for the primitive middle-dimensional cohomology of $\mathcal{X}_{L} \rightarrow \mathcal{S}_{L}$. Theorem 2.13 shows that one may compute the matrix $N(t)$ for the Gauss-Manin connection on $\mathcal{B}$. Theorem 3.13 then allows us to compute the pull back $\left(\mathcal{S}_{\mathcal{O}_{L}}\right)_{e} \rightarrow \mathcal{S}_{\mathcal{O}_{L}}$ and basis change matrix $H(s)$ needed so that the Picard-Fuchs matrix $N(s)^{\prime}$, say, with respect to the basis $\left(\mathcal{B}_{e}\right)_{[H(s)]}$ has a simple pole at $s=0$ with nilpotent residue matrix and converges on the punctured $p$-adic open unit disk. Let $-\delta$ be the minimum of the $p$-adic orders of $H(s)$ and $H(s)^{-1}$. Suppose that one can compute a $p^{N+2 \delta}$-approximation $\tilde{F}(t)$ to the matrix for Frobenius $F(t)$ on $\mathcal{B}_{\text {rig. }}$. Then by substituting $t=s^{e}$ and changing basis by $H(s)$ one obtains a $p^{N}$-approximation $\tilde{F}(s)^{\prime}$, say, to the matrix for Frobenius $F(s)^{\prime}$ on $\left(\mathcal{B}_{\text {rig, } e}\right)_{[H(s)]}$. By Lemma 3.2, the matrix $F(s)^{\prime}$ has no pole at $s=0$ and specialising $s N(s)^{\prime}$ and $\tilde{F}(s)^{\prime}$ at $s=0$ gives the data of a $p^{N}$-approximation of the limiting Frobenius structure. (One has to take some care in 'specialising' $\tilde{F}(s)^{\prime}$ at $s=0$, as this matrix may have a pole. To obtain an approximation of $F(0)^{\prime}$, embed the entries of $\tilde{F}(s)^{\prime}$ in $L((t))$ and take the coefficient of $t^{0}$.)

To compute the matrix $\tilde{F}(t)$ itself, we use the deformation method. This requires two inputs. First, the Picard-Fuchs matrix $N(t)$. Second, the matrix for the Frobenius action on the basis

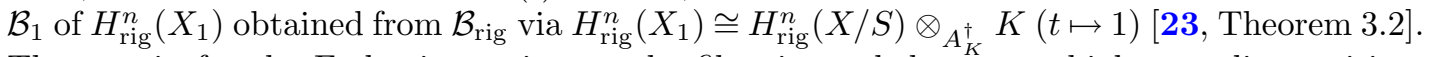
The matrix for the Frobenius action on the fibre is needed to some higher $p$-adic precision, due to precision loss during the deformation method. Precisions required can be calculated as in $[\mathbf{1 5}$, Section 6] and [23, Section 5]. One uses Kedlaya's algorithm to calculate the Frobenius matrix at the fibre $t=1[1, \S 3.5]$.

Note 3.16. Note that here Assumption (SP) is satisfied, since the primitive cohomology is just that of the family of complements of the smooth hypersurfaces in $\mathbb{P}_{W}^{n+1}$. Of course, by the primitive middle-dimensional limiting Frobenius structure we mean the limiting Frobenius structure in the middle dimension for this family of complements. Indeed, there is a small clash of notation between Sections 2 and 3 - we really wish to take the family ' $\mathcal{X} \rightarrow \mathcal{S}$ ' in Section 3 to be the family of complements of hypersurfaces, not the family ' $\mathcal{X}_{L} \rightarrow \mathcal{S}_{L}$ ' of hypersurfaces itself. 
3.3.2. Complexity. We do not attempt an asymptotic analysis of the algorithm in Theorem 3.15, but confine ourselves instead to a few comments.

Assume that the matrix for the Gauss-Manin connection with respect to the Dwork basis has a simple pole with nilpotent residue matrix. Then our algorithm is exactly the same as the original deformation method, except that we use the Frobenius lift $t \mapsto t^{p}$ rather than $t-1 \mapsto(t-1)^{p}$ (Note 3.3). When we solve the Picard-Fuchs differential system locally around $t=1$ during the algorithm, using the lift $t \mapsto t^{p}$ is slower in practice than using the lift $(t-1) \mapsto(t-1)^{p}$ (cf. $[\mathbf{2 3}, \S 5.1]$ ); however, provided that one uses the 'Tsuzuki method' [23, $\S 5.1 .2]$, the asymptotic complexity is the same. To obtain asymptotic complexity bounds, it is simpler to assume that one only computes the matrix for the connection with respect to the Dwork basis to a suitable $p$-adic precision rather than as an exact matrix of rational functions over an algebraic number field. This is all worked out explicitly in the analogous setting of 'Dwork cohomology' in [22, Sections 5, 6.1, 10, Step 2]. By comparison with the analysis in [23, Section 10], one sees that using an approximate connection matrix our algorithm certainly has running time which is polynomial in $p, \log _{p}(q),(d+1)^{n}$ and $N$.

A necessary condition for our assumption on the connection matrix to be met is that the pull back $e=1$, and hence the Picard-Lefschetz transformation, is unipotent, cf. Theorem 4.2. When this geometric condition is met (for example, a semistable degeneration of curves), the author has observed that frequently in practice our assumption on the connection matrix holds true. However, it is certainly not the case that it is always true, and the author does not understand its true geometric significance, if any.

When the matrix for the connection does not have a simple pole with nilpotent residue matrix with respect to the Dwork basis, the author makes no claims on the complexity of the algorithm. Computing pull backs and using shearing transformations to prepare eigenvalues is not too problematic - both were usually necessary for the examples in Section 5 . The most time-consuming problem in practice is when there is not even a simple pole, and one needs to use a cyclic vector; see Example 5.8.

\subsection{Limiting Frobenius-Hodge structures}

We conclude our description of degenerations in positive characteristic with a less formal discussion of the integral structure and Hodge filtration on our limiting Frobenius structures. Our hope is to convince the reader without detailed proofs that these additional structures exist and can be computed in quite wide generality.

Assume that we have an algebraic number field $L$ with ring of integers $\mathcal{O}_{L}$, and a smooth and proper morphism $\mathcal{X}_{\mathcal{O}_{L}} \rightarrow \mathcal{S}_{\mathcal{O}_{L}}$ of $\mathcal{O}_{L}$-schemes, where $\mathcal{S}_{\mathcal{O}_{L}}=\operatorname{Spec}\left(A_{\mathcal{O}_{L}}\right)$ with $A_{\mathcal{O}_{L}}:=\mathcal{O}_{L}[t, 1 / \Delta(t)]$ for some $\Delta(t) \in \mathcal{O}_{L}[t]$. We denote by $\mathcal{X}_{L} \rightarrow \mathcal{S}_{L}$ extension of scalars of $\mathcal{X}_{\mathcal{O}_{L}} \rightarrow \mathcal{S}_{\mathcal{O}_{L}}$ from $\mathcal{O}_{L}$ to $L$. We follow our usual convention of using the subscript $e$ to denote pulling back along the base via $s^{e}:=t$. Theorem 2.4 guarantees the existence of $e \geqslant 1$ and a basis for $H_{d R}^{m}\left(\left(\mathcal{X}_{L}\right)_{e} /\left(\mathcal{S}_{L}\right)_{e}\right)$ such that the matrix for the Gauss-Manin connection with respect to this basis has a simple pole at $s=0$ with nilpotent residue matrix. To simplify notation in our discussion, let us suppose $e=1$, and write $\mathcal{B}$ for the basis.

Let $p$ be a rational prime which is inert in $\mathcal{O}_{L}$, and $\mathcal{X} \rightarrow \mathcal{S}$ be the morphism obtained by base change from $\mathcal{O}_{L}$ to $W$. Denote by $H_{\text {cris }}^{m}(\mathcal{X} / \mathcal{S})$ relative crystalline cohomology. Write $A:=A_{\mathcal{O}_{L}} \otimes_{\mathcal{O}_{L}} W$, let $\hat{A}$ be the $p$-adic completion of $A$ and let $A^{\dagger}$ be the weak completion of $A$ (not tensored by $K$ ); see $\S 3.1$. We claim then that $\mathcal{B}$ maps naturally to a free $\hat{A}$-basis $\mathcal{B}_{\text {cris }}$ for $H_{\text {cris }}^{m}(\mathcal{X} / \mathcal{S})$ provided that $p$ is sufficiently large. We argue as follows.

For almost all such rational primes $p$, one may canonically map $\mathcal{B}$ to a subset $\mathcal{B}_{d R}$ of $H_{d R}^{m}(\mathcal{X} / \mathcal{S})$ and provided that $p$ is sufficiently large (depending on the geometry of the morphism $\left.\mathcal{X}_{L} \rightarrow \mathcal{S}_{L}\right)$ then $H_{d R}^{m}(\mathcal{X} / \mathcal{S})$ will be free with $\mathcal{B}_{d R}$ as an $A$-basis. One expects a crystalline relative comparison theorem, $H_{\text {cris }}^{m}(\mathcal{X} / \mathcal{S}) \cong H_{d R}^{m}(\mathcal{X} / \mathcal{S}) \otimes_{A} \hat{A}$, although the author does not know of a 
reference. Thus, extending scalars $\mathcal{B}_{d R}$ maps to an $\hat{A}$-basis $\mathcal{B}_{\text {cris }}$ of $H_{\text {cris }}^{m}(\mathcal{X} / \mathcal{S})$. One can make this construction of $\mathcal{B}_{\text {cris }}$ entirely explicit for families of smooth hypersurfaces using a relative version of the theorems and algorithms in [1].

Write $\mathcal{H}_{W}:=H_{\text {cris }}^{m}(\mathcal{X} / \mathcal{S})$ and denote by $\mathcal{F}$ the Frobenius on $\mathcal{H}_{W}$. The cohomology group $\mathcal{H}_{W}$ inherits a filtration $\mathcal{H}_{W}^{\bullet}$ from the Hodge filtration on $H_{d R}^{m}(\mathcal{X} / \mathcal{S})$. Applying Mazur's 'Frobenius-Hodge theorem' [27] fibre-by-fibre, it follows that $\mathcal{F}$ is 'divisible by $p^{i}$ ' on $\mathcal{H}_{W}^{i}$; that is, $\mathcal{F}\left(\mathcal{H}_{W}^{i}\right) \subseteq p^{i} \mathcal{H}_{W}$.

Assume now that $p$ is such that $\mathcal{B}_{\text {cris }}$ is a basis for $\mathcal{H}_{W}$. Let $F(t)_{\text {cris }}$ denote the matrix for $\mathcal{F}$ with respect to the basis $\mathcal{B}_{\text {cris }}$. Since $\mathcal{B}_{\text {cris }}$ is also naturally a basis for $H_{\text {rig }}^{m}(X / S)$, the matrix $F(t)_{\text {cris }}$ has entries in $A_{K}^{\dagger} \cap \hat{A}=A^{\dagger}$. The connection matrix $N(t)_{\text {cris }}$ has coefficients in $A_{\mathcal{O}_{L}} \subset A$ and a simple pole at $t=0$ with nilpotent residue matrix. For all but finitely many primes, $N(t)_{\text {cris }}$ will converge on the punctured $p$-adic open unit disk around zero. Thus, if we further exclude this finite set of primes, the basis $\mathcal{B}_{\text {cris }}$ will satisfy the hypotheses of Definition 3.4 and one can specialise at $t=0$ to obtain a triple $\left(\mathcal{H}_{0}, \mathcal{N}_{0}, \mathcal{F}_{0}\right)_{W}$ of a free $W$-module $\mathcal{H}_{0}$ with operators $\mathcal{N}_{0}$ and $\mathcal{F}_{0}$ such that $\mathcal{N}_{0} \mathcal{F}_{0}=p \mathcal{F}_{0} \mathcal{N}_{0}$ and $\mathcal{N}_{0}^{m+1}=0$. Moreover, there is a natural filtration $\mathcal{H}_{0}^{\bullet}$ such that $\mathcal{F}_{0}\left(\mathcal{H}_{0}^{i}\right) \subseteq p^{i} \mathcal{H}_{0}$ coming from the Hodge filtration on $\mathcal{H}_{W}$.

Allowing once again $e>1$, we have constructed the 'limiting Frobenius-Hodge structure' $\left(\mathcal{H}_{0}^{\bullet}, \mathcal{N}_{0}, \mathcal{F}_{0}, e\right)_{W}$ from Section 1. Note that when $\mathcal{X}_{L} \rightarrow \mathcal{S}_{L}$ 'extends' to a semistable degeneration over the origin (cf. Definition 4.9), we have $e=1$, and the pair ' $\left(\mathcal{H}_{0}^{\bullet}, \mathcal{N}_{0}\right)_{W} \otimes_{W} \mathbb{C}$ ' is exactly the limiting mixed Hodge structure from complex algebraic geometry $[\mathbf{3 2}$, Chapter 11].

Note 3.17. The term 'limiting mixed Hodge structure' arises naturally, since there are already the notions of a 'mixed Hodge structure' and a 'Hodge structure'. In rigid or crystalline cohomology, there is no formal notion of a 'Frobenius structure' to the author's knowledge, although the term is used loosely, for example in 'differential equation with a Frobenius structure'. The object which we call a 'limiting Frobenius structure' might be better called a 'limiting mixed $F$-isocrystal', and our 'limiting Frobenius-Hodge structure' a 'limiting mixed Hodge $F$-crystal'.

\section{Degenerations and 'Clemens-Schmidt'}

This section contains no original theorems, but rather gives a conjectural interpretation of the geometric significance of the limiting Frobenius structures we define. The notation in $\S \S 4.1$, 4.2.1 and 4.2.2 mainly follows the original sources, and only in $\S 4.2 .3$ do we return to the notation from Section 3 .

\subsection{Complex algebraic varieties}

The material in this section is based upon the exposition in [30]. Throughout, $H^{m}(\bullet)$ and $H_{m}(\bullet)$ denote singular cohomology and homology, respectively, with rational coefficients.

Let $\Delta$ denote the complex open unit disk. A degeneration is a proper flat holomorphic map $\pi: X \rightarrow \Delta$ of relative dimension $n$ such that $X_{t}:=\pi^{-1}(t)$ is a smooth complex variety for $t \neq 0$, and $X$ is a Kähler manifold. A degeneration is semistable if the central fibre $X_{0}$ is a reduced divisor with global normal crossings; that is, writing $X_{0}=\sum_{i} X_{0, i}$ as a sum of irreducible components, each $X_{0, i}$ is smooth and the $X_{0, i}$ meet transversally.

We recall a fundamental theorem on degenerations, due to Mumford with the assistance of Knudsen and Waterman; see [30] or [29, p. 102].

Theorem 4.1 (Semistable reduction). Given a degeneration $\pi: X \rightarrow \Delta$, there exist a base change $b: \Delta \rightarrow \Delta$ (defined by $t \mapsto t^{e}$ for some positive integer $e$ ), a semistable degeneration 
$\psi: Y \rightarrow \Delta$ and a diagram

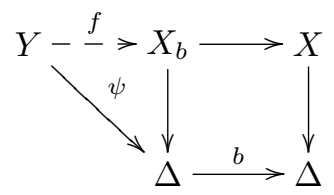

such that $f: Y \rightarrow X_{b}$ is a bimeromorphic map obtained by blowing up and blowing down subvarieties of the central fibre.

Let $\pi: X \rightarrow \Delta$ be a degeneration, and $\pi^{*}: X^{*} \rightarrow \Delta^{*}$ the restriction to the punctured disk $\Delta^{*}:=\Delta \backslash\{0\}$. Fix a smooth fibre $X_{t}(t \neq 0)$. Since $\pi^{*}$ is a $C^{\infty}$ fibration, the fundamental group $\pi_{1}\left(\Delta^{*}\right)$ acts on the cohomology $H^{m}\left(X_{t}\right)$ for each $0 \leqslant m \leqslant 2 n$. The map

$$
T_{m}: H^{m}\left(X_{t}\right) \rightarrow H^{m}\left(X_{t}\right)
$$

induced by the canonical generator of $\pi_{1}\left(\Delta^{*}\right)$ is called the Picard-Lefschetz or local monodromy transformation.

We have the following theorem, which is usually attributed independently to Griffiths, Grothendieck and Landman. (See [29, p. 106] for all but the claim on $k$, and [20, Theorem $\left.\mathrm{I}^{\prime}\right]$ for the claim on $k$.)

THEOREM 4.2 (Local monodromy theory). The local monodromy transformation $T_{m}$ is quasi-unipotent, with index of unipotency at most $m$. In other words, there is some $k$ such that

$$
\left(T_{m}^{k}-I\right)^{m+1}=0
$$

Moreover, if the central fibre $X_{0}$ is a (possibly non-reduced) divisor $\sum_{i} k_{i} X_{0, i}$ with the $X_{0, i}$ smooth hypersurfaces on $X$ meeting transversally, then one may take $k=\operatorname{lcm}\left\{k_{i}\right\}$. In particular, if $\pi: X \rightarrow \Delta$ is semistable, then $T$ is unipotent $(k=1)$.

Note that by blowing up subvarieties of the central fibre, one may always replace the original degeneration with one in which the central fibre is a (possibly non-reduced) union of smooth hypersurfaces crossing transversally without changing the family outside of the central fibre.

Let $\pi: X \rightarrow \Delta$ be a semistable degeneration of fibre dimension $n$, and $T_{m}$ the local monodromy transformation on $H^{m}\left(X_{t}\right)$ for some $0 \leqslant m \leqslant 2 n$. Since here the monodromy is unipotent, we can define the logarithm $H^{m}\left(X_{t}\right) \stackrel{N_{m}}{\longrightarrow} H^{m}\left(X_{t}\right)$ by the finite sum

$$
N_{m}:=\log \left(T_{m}\right)=\left(T_{m}-I\right)-\frac{1}{2}\left(T_{m}-I\right)^{2}+\frac{1}{3}\left(T_{m}-I\right)^{3}-\ldots
$$

The homology and cohomology groups with rational coefficients of the central fibre $X_{0}$ and the chosen fibre $X_{t}(t \neq 0)$ carry canonical mixed Hodge structures [30, pp. 114-116]. These fit together in an elaborate exact sequence.

THEOREM 4.3 (Clemens-Schmidt sequence). Let $\pi: X \rightarrow \Delta$ be a semistable degeneration and $N_{m}$ the logarithm of local monodromy on $H^{m}\left(X_{t}\right)$ for some $t \neq 0$. There exists an exact sequence of mixed Hodge structures

$$
\begin{gathered}
\quad \ldots \rightarrow H_{2 n+2-m}\left(X_{0}\right) \rightarrow H^{m}\left(X_{0}\right) \rightarrow H^{m}\left(X_{t}\right) \stackrel{N_{m}}{\longrightarrow} H^{m}\left(X_{t}\right) \\
\rightarrow H_{2 n-m}\left(X_{0}\right) \rightarrow H^{m+2}\left(X_{0}\right) \rightarrow H^{m+2}\left(X_{t}\right) \stackrel{N_{m+2}}{\longrightarrow} H^{m+2}\left(X_{t}\right) \rightarrow \ldots
\end{gathered}
$$

All the maps in this exact sequence are described explicitly in [30, pp. 108-109]. 


\subsection{Varieties over finite fields}

The material in $\S \S 4.2 .1$ and 4.2.2 follows closely the presentation in [12]. Beware that the ' $K$ ' in these sections need not be the $p$-adic field from Section 3. Indeed, we are interested in geometric rather than arithmetic degenerations, and so in our application $K$ is equicharacteristic.

4.2.1. Semistable reduction. Let $R$ be a henselian discrete valuation ring with field of fractions $K$ and residue field $k$ of characteristic $p>0$. Choose an algebraic closure $\bar{K}$ of $K$, and denote by $\bar{R}$ the normalisation of $R$ in $\bar{K}$, and $\bar{k}$ the residue field of $\bar{R}$ (which is an algebraic closure of $k$ ).

Let $S:=\operatorname{Spec}(R), s:=\operatorname{Spec}(k), \bar{s}:=\operatorname{Spec}(\bar{k}), \eta:=\operatorname{Spec}(K), \bar{\eta}:=\operatorname{Spec}(\bar{K})$ and $f: X \rightarrow S$ be a proper morphism. We say that $f$ is semistable if locally for the étale topology $X$ is $S$ isomorphic to $R\left[t_{1}, \ldots, t_{n}\right] /\left(t_{1} \ldots t_{r}-\pi\right)$, where $\pi$ is a uniformiser on $R$. This implies that the generic fibre $X_{\eta}$ is smooth, $X$ is regular and the special fibre $X_{s}$ is a divisor with normal crossings on $X$.

The following fundamental conjecture is known to be true at least when $X_{\eta}$ is a smooth projective curve.

Conjecture 4.4 (Semistable reduction). If $X_{\eta}$ is smooth over $\eta$, then there exists a finite extension $\eta^{\prime}$ of $\eta$ such that $X_{\eta^{\prime}}$ admits a proper and semistable model $X^{\prime} \rightarrow S^{\prime}$ on the normalisation $S^{\prime}$ of $S$ in $\eta^{\prime}$.

4.2.2. Local monodromy in étale cohomology. Let $I$ be the inertia group, defined by the exact sequence

$$
1 \rightarrow I \rightarrow \operatorname{Gal}(\bar{K} / K) \rightarrow \operatorname{Gal}(\bar{k} / k) \rightarrow 1 .
$$

Write $G:=\operatorname{Gal}(\bar{K} / K)$ and let $\rho: G \rightarrow \operatorname{GL}(V)$ be a continuous representation of $G$ to a finitedimensional $\mathbb{Q}_{\ell^{-}}$-vector space $(\ell \neq p)$. We say that the representation is quasi-unipotent if there is an open subgroup $I_{1}$ of $I$ such that the restriction to $I_{1}$ of $\rho$ is unipotent, that is, $\rho(g)$ is unipotent for all $g \in I_{1}$.

Let $f: X \rightarrow S$ be a proper morphism, and $H^{m}\left(X_{\bar{\eta}}, \mathbb{Q}_{\ell}\right)$ denote étale cohomology with coefficients in $\mathbb{Q}_{\ell}$ of $X_{\bar{\eta}}$. Then there exists a local monodromy representation

$$
\rho_{m}: G \rightarrow \operatorname{GL} H^{m}\left(X_{\bar{\eta}}, \mathbb{Q}_{\ell}\right) .
$$

We have the following analogues of Theorem 4.2.

Theorem $4.5\left[\mathbf{1 2}\right.$, Corollaire 3.3]. The local monodromy representation on $H^{m}\left(X_{\bar{\eta}}, \mathbb{Q}_{\ell}\right)$ for a semistable degeneration $f: X \rightarrow S$ is unipotent. More precisely, for $g \in I$, we have $\left(\rho_{m}(g)-1\right)^{m+1}=0$.

Corollary 4.6 [12, Corollaire 3.4]. Let $f: X \rightarrow S$ be a proper morphism with $X_{\eta}$ smooth over $\eta$. Assuming geometric semistable reduction (Conjecture 4.4), the local monodromy representation on $H^{m}\left(X_{\bar{\eta}}, \mathbb{Q}_{\ell}\right)$ is quasi-unipotent.

Note 4.7. Grothendieck proved that any $\ell$-adic representation of $G$ is quasi-unipotent, under a mild hypothesis on $k$ [12, Théorème 1.2]. In the case in which $k$ is a finite field, this gives a proof of Corollary 4.6 which does not need geometric semistable reduction.

Let $f: X \rightarrow S$ be a semistable degeneration and $\rho_{m}$ the associated monodromy representation (4.1). There exist a homomorphism $t_{\ell}: I \rightarrow \mathbb{Z}_{\ell}(1)$ and a unique nilpotent morphism

$$
N_{m}: H^{m}\left(X_{\bar{\eta}}, \mathbb{Q}_{\ell}\right)(1) \rightarrow H^{m}\left(X_{\bar{\eta}}, \mathbb{Q}_{\ell}\right)
$$


such that

$$
\rho_{m}(g)=\exp \left(N_{m} t_{\ell}(g)\right) \quad \text { for all } g \in I .
$$

Assume now that $k$ is finite, and $S$ is the henselisation of a closed point on a smooth curve over $k$. (These conditions ensure in particular that the weight-monodromy conjecture holds true; see [12, pp. 41-42].) The following is the analogue in $\ell$-adic étale cohomology of one piece of the Clemens-Schmidt sequence.

Theorem 4.8 (Local invariant cycle theorem [12, Corollaire 3.11]). For all $m$, there exists an exact sequence

$$
H^{m}\left(X_{\bar{s}}, \mathbb{Q}_{\ell}\right) \rightarrow H^{m}\left(X_{\bar{\eta}}, \mathbb{Q}_{\ell}\right) \stackrel{N_{m}}{\longrightarrow} H^{m}\left(X_{\bar{\eta}}, \mathbb{Q}_{\ell}\right)(-1)
$$

4.2.3. Rigid cohomology. There is not such a coherently worked out picture for the rigid (or crystalline) cohomology of a degeneration. See [2, 28] for some partial results for rigid cohomology (and the references therein for crystalline cohomology). By analogy though, the following suggests itself.

Let $X \rightarrow S \subseteq \mathbb{A}_{\mathbb{F}_{q}}^{1}$ be a morphism of varieties over $\mathbb{F}_{q}$ which has a good lift around $t=0$ (Definition 3.10). Then the limiting Frobenius structure $\left(\mathcal{H}_{0}, \mathcal{N}_{0}, \mathcal{F}_{0}, e\right)$ for $\mathcal{X} \rightarrow \mathcal{S}$ in dimension $m$ around $t=0$ exists and is unique (Theorem 3.8 and Lemma 3.6). We shall write $\left(\mathcal{H}_{0}^{m}, \mathcal{N}_{0}, \mathcal{F}_{0}, e_{m}\right)$ when we wish to indicate the dependence of the limiting Frobenius structure on $m$.

Recall here that $\mathcal{S}=\operatorname{Spec}(W[t, 1 / \Delta(t)])$ for some polynomial $\Delta(t)$ which has no zeros in $\bar{K}$ within the punctured $p$-adic open unit disk around zero but may vanish at $t=0$. It is interesting to note that if $\Delta(0)=0 \bmod p$ (that is, $0 \notin S$ ), then we must have $\Delta(0)=0$. (This follows by looking at Newton polygons, since $\Delta(t)$ is not identically zero modulo $p$ (for otherwise $S$ is empty) and therefore $\Delta(t)$ contains a root in the $p$-adic open unit disk.)

Definition 4.9. Let $X \rightarrow S$ be any smooth morphism of varieties over $\mathbb{F}_{q}$, where $S \subseteq$ $\mathbb{A}_{\mathbb{F}_{q}}^{1} \backslash\{0\}$. Let $T:=S \cup\{0\}$ be the variety obtained by adjoining the origin to $S$. We shall say that $X \rightarrow S$ extends to a semistable degeneration over the origin if there exists a semistable degeneration $Y \rightarrow T$ which restricts to $X \rightarrow S$ over $S$.

Here by 'semistable degeneration' we mean in the sense of $\S 4.2 .1$ when one localises $Y \rightarrow T$ around the origin. In this situation, we shall write $X_{0}$ for the fibre of $Y \rightarrow T$ at the origin and refer to it as the degenerate fibre. Note $X \rightarrow S$ may extend to a semistable degeneration over the origin in more than one way; for example, one can blow up smooth points on the degenerate fibre. Let us also define a semistable $\mathbb{F}_{q}$-variety as one which is étale locally isomorphic to $\mathbb{F}_{q}\left[t_{1}, \ldots, t_{n}\right] /\left(t_{1} \ldots t_{r}\right)$ for some $n$ and $r \leqslant n$; so the degenerate fibre in a semistable degeneration is semistable.

We recall the definition of a basic object in rigid cohomology.

Definition 4.10. An $F$-isocrystal $M$ over $K$ is a finite-dimensional $K$-vector space equipped with an injective $\sigma$-linear map $F$. For $m \in \mathbb{Z}$, define $M(m)$ to be the $F$-isocrystal with the same underlying vector space as $M$ but with the action of $F$ multiplied by $p^{-m}$.

The $F$-isocrystal associated to the limiting Frobenius structure $\left(\mathcal{H}_{0}^{m}, \mathcal{N}_{0}, \mathcal{F}_{0}, e_{m}\right)$ is the pair $\left(\mathcal{H}_{0}^{m}, \mathcal{F}_{0}\right)$.

Define $e:=\operatorname{lcm}_{0 \leqslant m \leqslant 2 n}\left(e_{m}\right)$, where $n$ is the fibre dimension of $X \rightarrow S$. One may pull back the base $\mathcal{X}_{e} \rightarrow \mathcal{X}_{e_{m}}$ and the 'smallest' limiting Frobenius structure $\left(\mathcal{H}_{0}^{m}, \mathcal{N}_{0}, \mathcal{F}_{0}, e_{m}\right)$ in dimension $m$ is replaced by $\left(\mathcal{H}_{0}^{m},\left(e / e_{m}\right) \mathcal{N}_{0}, \mathcal{F}_{0}, e\right)$. Note that this has no effect on the kernel 
and cokernel of the monodromy operator, since $\mathcal{N}_{0}$ is multiplied by a non-zero integer, that is, there is no ambiguity in replacing $e_{m}$ by $e$.

Conjecture 4.11. Let $X \rightarrow S \subseteq \mathbb{A}_{\mathbb{F}_{q}}^{1} \backslash\{0\}$ be a morphism of varieties over $\mathbb{F}_{q}$ which has a good lift around $t=0$ and denote by $\left(\mathcal{H}_{0}^{m}, \mathcal{N}_{0}, \mathcal{F}_{0}, e_{m}\right)$ the limiting Frobenius structure at $t=0$ in dimension $m$. Define $e:=\operatorname{lcm}_{0 \leqslant m \leqslant 2 n}\left(e_{m}\right)$. Then the morphism $X_{e} \rightarrow S_{e}$ obtained by pulling back the base $S_{e} \rightarrow S$ extends to a semistable degeneration over the origin with degenerate fibre $X_{e, 0}$. Moreover, for any such extension there exists a 'Clemens-Schmidt' exact sequence of F-isocrystals

$$
\begin{gathered}
\quad \ldots \rightarrow H_{2 n+2-m, \text { rig }}\left(X_{e, 0}\right)(-n-1) \rightarrow H_{\text {rig }}^{m}\left(X_{e, 0}\right) \rightarrow \mathcal{H}_{0}^{m} \stackrel{\mathcal{N}_{0}}{\longrightarrow} \mathcal{H}_{0}^{m}(-1) \\
\rightarrow H_{2 n-m, \text { rig }}\left(X_{e, 0}\right)(-n-1) \rightarrow H_{\text {rig }}^{m+2}\left(X_{e, 0}\right) \rightarrow \mathcal{H}_{0}^{m+2} \stackrel{\mathcal{N}_{0}}{\longrightarrow} \mathcal{H}_{0}^{m+2}(-1) \rightarrow \ldots
\end{gathered}
$$

for some covariant 'rigid homology' functor $H_{j, \text { rig }}(\bullet)$ on semistable $\mathbb{F}_{q}$-varieties which vanishes outside of dimensions $0 \leqslant j \leqslant 2 n$.

The twistings here are chosen to be consistent with those on the filtered vector spaces [30, pp. 108-109], and of course the equation $\mathcal{N}_{0} \mathcal{F}_{0}=p \mathcal{F}_{0} \mathcal{N}_{0}$. (The referee pointed out that rigid homology for arbitrary $\mathbb{F}_{q}$-varieties is developed in [33, Section 2]: it is defined as the dual of rigid cohomology with compact support. Moreover, motivated by the first version of this paper, Chiarellotto and Tsuzuki outlined to the author the construction of a Clemens-Schmidt sequence for semistable degenerations in rigid cohomology, and explained how one should be able to prove exactness using results due to Crew [6, Theorem 10.8]. A description of this is beyond the scope of this paper, but the author hopes it may be fully worked out and written up.)

For $n=1$ and $m$ odd, our 'Clemens-Schmidt' sequence is

$$
0 \rightarrow H_{\text {rig }}^{1}\left(X_{e, 0}\right) \rightarrow \mathcal{H}_{0}^{1} \stackrel{\mathcal{N}_{0}}{\longrightarrow} \mathcal{H}_{0}^{1}(-1) \rightarrow H_{1, \text { rig }}\left(X_{e, 0}\right)(-2) \rightarrow 0 .
$$

Thus, according to Conjecture 4.11, the polynomial

$$
\operatorname{det}\left(1-T \mathcal{F}_{0}^{\log _{p}(q)} \mid \operatorname{Ker}\left(\mathcal{N}_{0}\right)\right)
$$

is the numerator of the zeta function of the semistable curve $X_{e, 0}$. (The author is implicitly assuming here the existence of a trace formula for semistable curves, cf. (1.1).) One can test this for smooth plane curves by combining our algorithm for computing limiting Frobenius structures of hypersurfaces with explicit ad hoc computations of 'stable limits' of plane curves.

For $n>1$, our 'Clemens-Schmidt' sequence would involve in a non-trivial manner the unknown 'rigid homology' groups $H_{j, \text { rig }}\left(X_{e, 0}\right)$. It seems reasonable to conjecture though that the degenerate fibre $X_{e, 0}$ arises by reduction modulo $p$ from a $W$-scheme $\mathcal{X}_{e, 0}$ and dropping the Frobenius structure we have an isomorphism of $K$-vector spaces

$$
H_{j, \text { rig }}\left(X_{e, 0}\right) \cong H_{j}\left(\left(\mathcal{X}_{e, 0}\right)_{K}\right)
$$

where $\left(\mathcal{X}_{e, 0}\right)_{K}$ is the generic fibre and $H_{j}(\bullet)$ singular homology with coefficients in $K$. Thus, under this further conjecture, the dimensions at least of our unknown spaces are amenable to computation.

\section{Examples}

In this section, we present some explicit examples of degenerations of curves, surfaces and threefolds with the aim of illustrating our construction and providing evidence in support of Conjecture 4.11. We follow here the paradigm from experimental science rather than mathematics. That is, we test experimentally consequences of our conjecture and if there is no inconsistency declare we have found evidence in support of it. 


\subsection{Preparation}

We first discuss our choice of examples, and explain exactly what we computed in each case.

5.1.1. The families. In our examples, the family $X \rightarrow S$ is defined by an equation

$$
P_{t}:=(1-t) P_{0}+t P_{1}=0
$$

for specific choices of homogeneous polynomials $P_{0}, P_{1} \in \mathbb{F}_{p}\left[x_{0}, \ldots, x_{n+1}\right]$. We write the variables $x_{0}, x_{1}, \ldots, x_{n+1}$ as $X, Y, Z$ and so on. The good algebraic lift $\mathcal{X}_{\mathbb{Z}} \rightarrow \mathcal{S}_{\mathbb{Z}}$ (Definition 3.14) of our family is defined by the same equation only with the polynomials $P_{0}$ and $P_{1}$ thought of as having integer coefficients. (See the first paragraph of $\S 3.3 .1$ for the exact construction of $\mathcal{X}_{\mathbb{Z}} \rightarrow \mathcal{S}_{\mathbb{Z}}$ from the polynomial $P_{t}$; here we take $\Delta(t)$ to be $\Delta_{n, d}\left(P_{t}\right)$.)

Our algorithm outputs a suitable approximation to the limiting Frobenius structure $\left(\mathcal{H}_{0}, \mathcal{N}_{0}, \mathcal{F}_{0}, e\right)$ at $t=0$ for the primitive middle-dimensional cohomology of $X \rightarrow S$; cf. Theorem 3.15. By suitable approximation, we mean here a $p^{N}$-approximation for $N$ sufficiently large in each example for the author to identify a plausible integer polynomial $\operatorname{det}\left(1-T \mathcal{F}_{0} \mid \mathcal{H}_{0}\right)$. We do not claim that this polynomial is provably correct; however, our choice of $N$ is based upon expectations on the size of the coefficients from the weight-monodromy conjecture (§ 5.1.2) and consideration of the $\mathbb{Z}_{p}$-lattice structure and Hodge filtration on the limiting Frobenius structure $(\S 3.4$ and $[\mathbf{2 3}, \S 9.3 .2])$.

5.1.2. The weight-monodromy conjecture. The nilpotent monodromy operator $\mathcal{N}_{0}$ defines an increasing filtration

$$
\{0\}=:\left(\mathcal{H}_{0}\right)_{-1} \subseteq\left(\mathcal{H}_{0}\right)_{0} \subseteq\left(\mathcal{H}_{0}\right)_{1} \subseteq \ldots \subseteq\left(\mathcal{H}_{0}\right)_{2 n}:=\mathcal{H}_{0}
$$

on the $\mathbb{Q}_{p}$-vector space $\mathcal{H}_{0}[\mathbf{2 9}$, pp. 106-107]. The filtration is defined as a 'convolution' of the two filtrations $\left(\operatorname{Ker} \mathcal{N}_{0}^{k}\right)_{k=0}^{n+1}$ and $\left(\operatorname{Im} \mathcal{N}_{0}^{i}\right)_{i=0}^{n+1}[\mathbf{1 2},(1.5 .5)]$. Since $\mathcal{N}_{0} \mathcal{F}_{0}=p \mathcal{F}_{0} \mathcal{N}_{0}$, the filtration is therefore stabilised by the Frobenius $\mathcal{F}_{0}$. In the case of curves $(n=1)$, we have $\mathcal{N}_{0}^{2}=0$ and the filtration is

$$
\{0\} \subseteq \operatorname{Im}\left(\mathcal{N}_{0}\right) \subseteq \operatorname{Ker}\left(\mathcal{N}_{0}\right) \subseteq \mathcal{H}_{0} .
$$

For surfaces $(n=2)$, we have $\mathcal{N}_{0}^{3}=0$ and here the filtration is

$$
\{0\} \subseteq \operatorname{Im}\left(\mathcal{N}_{0}^{2}\right) \subseteq \operatorname{Im}\left(\mathcal{N}_{0}\right) \cap \operatorname{Ker}\left(\mathcal{N}_{0}\right) \subseteq \operatorname{Im}\left(\mathcal{N}_{0}\right)+\operatorname{Ker}\left(\mathcal{N}_{0}\right) \subseteq \operatorname{Ker}\left(\mathcal{N}_{0}^{2}\right) \subseteq \mathcal{H}_{0} .
$$

Note that for surfaces when $\mathcal{N}_{0}^{2}=0$ then (5.3) becomes (5.2), only with indices shifted by one. In any dimension $n$, we have $\mathcal{N}_{0}^{n+1}=0$ (Theorem 4.2), but when in fact $\mathcal{N}_{0}=0$ the filtration then becomes

$$
\left(\mathcal{H}_{0}\right)_{n-1}:=\{0\} \subseteq \mathcal{H}_{0}=:\left(\mathcal{H}_{0}\right)_{n} .
$$

These cases cover the ones we shall encounter in our examples.

For $j$ such that $2 j \in \mathbb{Z}$ and $Q(T) \in 1+T \mathbb{Q}[T]$, we shall call $Q(T)$ a weight $j$ Weil polynomial with respect to $p$ if all of its reciprocal roots have complex absolute value $p^{j}$. We shall say that the weight-monodromy conjecture holds for our construction if for each $0 \leqslant j \leqslant 2 n$, we have $\operatorname{det}\left(1-T \mathcal{F}_{0} \mid\left(\mathcal{H}_{0}\right)_{j} /\left(\mathcal{H}_{0}\right)_{j-1}\right)$ a weight $j / 2$ Weil polynomial with respect to $p$.

For each of our examples, we state the dimensions of the subspaces in the monodromy filtration, and we verified that the weight-monodromy conjecture holds for our construction. This gives evidence for the first part of Conjecture 4.11, in the following slightly loose sense. If the morphism $X_{e} \rightarrow S_{e}$ does extend to a semistable degeneration over the origin, then the weight-monodromy conjecture holds in the setting of $\ell$-adic étale cohomology [12, pp. 41-42]. So, one at least expects that it should hold for our construction, although the author does not offer a proof of this. 
We note that for an open smooth $W$-scheme a comparison theorem between a filtration defined by Frobenius 'weights' in the rigid cohomology of the special fibre and the weight filtration in the algebraic de Rham cohomology of the generic fibre was proved in [3].

5.1.3. Stable limits of curves. The subspace $\left(\mathcal{H}_{0}\right)_{2 n-1}$ in the filtration for curves $(n=1)$ is $\operatorname{Ker} \mathcal{N}_{0}$. We write down $Q(T):=\operatorname{det}\left(1-T \mathcal{F}_{0} \mid \operatorname{Ker} \mathcal{N}_{0}\right)$ for our examples of curve degenerations. According to Conjecture 4.11, after pulling back $s^{e}:=t$ our degeneration should extend to a semistable degeneration over the origin with degenerate fibre the curve $X_{e, 0}$ and $Q(T)$ should be the numerator of the zeta function of $X_{e, 0}$. We provide evidence in support of this in $\S 5.2 .1$, using the recipes in [11] to construct at least geometrically the 'stable limits' of our families at the origin. Writing $X_{0}$ for the fibre at $t=0$ in our original family, defined by the polynomial $P_{0}$, it is amusing to note that $Q(T)$ can have nothing whatsoever to do with the zeta function of the singular fibre $X_{0}$ itself.

Note 5.1. In [18], Kloosterman gives an interesting discussion of the application of both the deformation method and Kedlaya's algorithm to singular projective hypersurfaces. Several low-degree examples in which the cohomology is pure 'algebraic' are presented. It is not clear to the present author that the methods based on deformation in that paper can be generally applied; for example, the lift of Frobenius chosen may cause difficulties, cf. Note 3.3. However, the idea that some appropriate version of the deformation method should compute the stable limit of a degeneration of curves is due to Kloosterman.

5.1.4. Comments on implementation. Our choice of examples was limited in several ways. First, it was convenient to assume that the degree $d$ divides $p-1$ and take $P_{1}:=$ $x_{0}^{d}+\ldots+x_{n+1}^{d}$, so that we could easily write down the Frobenius matrix at $t=1$, cf. $[\mathbf{1 5}$, $\S 1.5]$. Second, we restricted attention mainly to examples in which the Picard-Fuchs matrix with respect to the Dwork basis had only a simple pole at $t=0$ (and converged in the punctured $p$-adic open unit disk). This avoided having to use a cyclic vector, which we found time consuming. See Example 5.8 for an exception. Third, for the quartic surface examples we chose polynomials $P_{0}$ with few non-zero terms. This was needed for the Gröbner basis computation to finish within a reasonable time (see Note 2.9 for a method avoiding this). With these restrictions, we were still able to examine many different types of degenerations (some of which were suggested to the author by Damiano Testa).

The experiments were carried out using a machine with four quad-core processors $(2.60$ $\mathrm{GHz}$ ) and $64 \mathrm{~GB}$ RAM. Approximate timings were: Example 5.2 (1 minute), Example 5.3 (3 minutes), Example 5.4 (19 hours), Example 5.5 (4 hours), Example 5.7 (44 hours), Example 5.8 (48 minutes), Example 5.9 (41 minutes), Example 5.10 (1 minute), Example 5.11 (95 minutes), Example 5.12 (2 hours), Example 5.13 (4 hours), Example 5.14 (11 hours) and Example 5.15 (6 hours). Space consumption for these examples varied accordingly up to around 6 GB RAM.

\subsection{The experiments}

We now present the results of our computational experiments.

5.2.1. Examples directly supporting Conjecture 4.11. In the following examples, we were able to compute geometrically the stable limit of the degeneration and provide strong evidence supporting Conjecture 4.11.

Example 5.2. Consider the degeneration (5.1) of a diagonal quartic curve to a double conic defined by taking $P_{1}:=X^{4}+Y^{4}+Z^{4}$ and

$$
P_{0}:=\left(X^{2}+Y^{2}+3 Z^{2}+3 X Y+Y Z+2 X Z\right)^{2} .
$$


Here $e=2$ and by pulling back the base via $s^{2}:=t$ we get the monodromy operator $\mathcal{N}_{0}=0$. The monodromy filtration (5.2) has spaces of dimensions $0,0,6,6$, respectively. For the prime $p:=5$, the reverse characteristic polynomial of the Frobenius $\mathcal{F}_{0}$ on $\operatorname{Ker}\left(\mathcal{N}_{0}\right)=\mathcal{H}_{0}$ is

$$
Q(T)=1-6 T+23 T^{2}-58 T^{3}+115 T^{4}-150 T^{5}+125 T^{6} .
$$

This is a weight $\frac{1}{2}$ Weil polynomial with respect to 5 . The zeta function of the fibre $X_{0}$ is $1 /(1-T)(1-5 T)$, which is not related to the polynomial $Q(T)$. However, the 'stable limit' $X_{2,0}$ of the family around zero is a hyperelliptic curve of genus two which is constructed as a double cover of the conic

$$
C:=X^{2}+Y^{2}+3 Z^{2}+3 X Y+Y Z+2 X Z=0
$$

ramified at the eight points of intersection of $C=0$ with $P_{1}=0$ [11, pp. 133-134]. By direct computation of these points and projection to an axis, one finds that over $\mathbb{F}_{5}$ the curve $X_{2,0}$ has equation

$$
y^{2}=a\left(x^{8}+3 x^{7}+2 x^{6}+4 x^{3}+x^{2}+2 x+1\right)
$$

for some non-zero $a \in \mathbb{F}_{5}$. Taking $a$ to be a non-square in $\mathbb{F}_{5}$ gives a curve whose zeta function is indeed $Q(T) /(1-T)(1-5 T)$. (For $a$ a square, the zeta function is $Q(-T) /(1-T)(1-5 T)$.) So, this computation is consistent with Conjecture 4.11 .

Observe that if we changed the polynomial $P_{1}$ in Example 5.2, then the stable limit of the degeneration would (usually) be different, since it depends upon the points of intersection of $P_{1}$ with the conic. Thus, the stable limit can depend upon the family, not just the degenerate fibre.

EXAMPLE 5.3. Consider the degeneration (5.1) of a diagonal quartic curve to a threecuspidal quartic (cusps $(0: 0: 1),(0: 1: 0),(1: 0: 0))$ defined by taking $P_{1}:=X^{4}+Y^{4}+Z^{4}$ and

$$
P_{0}:=X^{2} Y^{2}+Y^{2} Z^{2}+Z^{2} X^{2}-2 X Y Z(X+Y+Z) .
$$

Here $e=6$ and pulling back the base via $s^{6}:=t$ the local monodromy becomes trivial; that is, $\mathcal{N}_{0}=0$. So, as before, the monodromy filtration (5.2) consists of spaces of dimensions $0,0,6,6$, respectively. For the prime $p:=13$, the reverse characteristic polynomial of the Frobenius $\mathcal{F}_{0}$ on $\operatorname{Ker}\left(\mathcal{N}_{0}\right)=\mathcal{H}_{0}$ is

$$
Q(T)=\left(1-5 T+13 T^{2}\right)^{3} .
$$

This is a weight $\frac{1}{2}$ Weil polynomial with respect to 13 . The fibre $X_{0}$ has geometric genus zero and zeta function $1 /(1-T)(1-13 T)$. The 'stable limit' around zero for the family over $\mathbb{C}$ is a projective line $\mathbb{P}_{\mathbb{C}}^{1}$ and three non-intersecting copies of an elliptic curve $\mathcal{E}_{\mathbb{C}}$ with $j$-invariant zero each meeting the projective line transversely at one point; cf. [11, pp. 122-128], where the elaborate calculation of the stable limit of a curve acquiring a cusp is performed. The polynomial $1-5 T+13 T^{2}$ is the numerator of the zeta function of an elliptic curve $E:=\mathcal{E} \times_{\mathbb{Z}} \mathbb{F}_{13}$, where $\mathcal{E}_{\mathbb{C}}:=\mathcal{E} \times_{\mathbb{Z}} \mathbb{C}$ has $j$-invariant zero, for $\mathcal{E}$ some elliptic curve 'over $\mathbb{Z}$ ' (indeed, several). Thus, this is consistent with Conjecture 4.11 taking $X_{6,0}$ to be $\mathbb{P}_{\mathbb{F}_{13}}^{1} \cup E \cup E \cup E$ with appropriate intersections.

EXAmple 5.4. Consider the degeneration (5.1) of a diagonal quintic to a quintic with a triple point $(0: 0: 1)$ defined by taking $P_{1}:=X^{5}+Y^{5}+Z^{5}$ and

$$
P_{0}:=X^{5}+Y^{5}+\left(X^{4}+3 X^{3} Y+Y^{4}\right) Z+\left(2 X^{3}+X Y^{2}+3 Y^{3}\right) Z^{2} .
$$

Here $e=3$ and pulling back the base via $s^{3}:=t$ gives non-trivial nilpotent monodromy $\mathcal{N}_{0}$. We have $\mathcal{N}_{0}^{2}=0$ and the monodromy filtration (5.2) consists of spaces of dimensions $0,2,10,12$, 
respectively. For $p:=11$, the reverse characteristic polynomial of the Frobenius $\mathcal{F}_{0}$ acting on $\operatorname{Ker}\left(\mathcal{N}_{0}\right)$ is

$$
Q(T)=(1-T)(1+T)\left(1+11 T^{2}\right)\left(1+5 T+22 T^{2}+62 T^{3}+242 T^{4}+605 T^{5}+1331 T^{6}\right) .
$$

The latter degree eight factor is a weight $\frac{1}{2}$ Weil polynomial with respect to 11 . The 'stable limit' around zero for the family over $\mathbb{C}$ is the normalisation of the degenerate fibre meeting an elliptic curve with $j$-invariant zero at the three points lying over the triple point of the degenerate fibre [11, pp. 131-132]. The degree six factor in $Q(T)$ is the numerator of the zeta function of the normalisation, and $1+11 T^{2}$ the numerator of the zeta function of $E:=\mathcal{E} \times \mathbb{Z} \mathbb{F}_{11}$, where $\mathcal{E}_{\mathbb{C}}:=\mathcal{E} \times_{\mathbb{Z}} \mathbb{C}$ has $j$-invariant zero, for $\mathcal{E}$ some elliptic curve 'over $\mathbb{Z}$ '. So, this example supports Conjecture 4.11.

EXAmPle 5.5. Consider the degeneration (5.1) of a diagonal sextic to a sextic curve with an ordinary double point $(0: 0: 1)$ defined by taking $P_{1}:=X^{6}+Y^{6}+Z^{6}$ and

$$
P_{0}:=X^{6}+Y^{6}+3 Y^{4} Z^{2}+2 X^{3} Z^{3}+\left(X^{2}+Y^{2}\right) Z^{4} .
$$

Here $e=1$, and no pull back on the base was required, but the monodromy operator $\mathcal{N}_{0}$ is nonzero. The monodromy filtration (5.2) consists of spaces of dimensions $0,1,19,20$, respectively. For the prime $p:=7$, the reverse characteristic polynomial of the Frobenius $\mathcal{F}_{0}$ acting on $\operatorname{Ker}\left(\mathcal{N}_{0}\right)$ is

$$
\begin{aligned}
Q(T)= & (1+T)\left(1-T+7 T^{2}\right)\left(1+T-9 T^{3}+49 T^{5}+343 T^{6}\right) \\
& \times\left(1-4 T+19 T^{2}-60 T^{3}+179 T^{4}-522 T^{5}+1253 T^{6}\right. \\
& \left.-2940 T^{7}+6517 T^{8}-9604 T^{9}+16807 T^{10}\right) .
\end{aligned}
$$

The fibre $X_{0}$ is semistable, and since the total space is smooth we have a semistable degeneration. Indeed, $X_{1,0}:=X_{0}$ has zeta function $Q(T) /(1-T)(1-7 T)$ as predicted by Conjecture 4.11.

Note 5.6. One can illustrate in a simple manner how our method can be used to compute the local factors at 'bad primes' in the L-series of a curve using Example 5.5. Consider the plane curve over $\mathbb{Z}$ defined by the polynomial $R_{0}:=P_{0}+7 Z^{6}$ with $P_{0}$ the polynomial from Example 5.5 but thought of as having integer coefficients. Then this curve is smooth over $\mathbb{Q}$ but the reduction modulo the prime $p:=7$ is the singular sextic $X_{0}$. The local factor at $p:=7$ in the L-series of the curve defined by $R_{0}$ is $Q(T)$. Note here that one computes this local factor by lifting $R_{0} \bmod 7$ to the integer polynomial $P_{0}$, not the integer polynomial $R_{0}$ itself. The reason for this is that the family over $\mathbb{Q}$ defined by the equation $(1-t) R_{0}+t P_{1}$ is not smooth in the punctured $p$-adic open unit disk around $t=0$ : since $R_{0}$ defines a smooth curve over $\mathbb{Q}$ but $R_{0}$ mod 7 defines a singular curve over $\mathbb{F}_{7}$, this forces there to be a singular fibre in the family for some $t=t_{0}$ with $\operatorname{ord}_{7}\left(t_{0}\right)>0$ (in fact, $\operatorname{ord}_{7}\left(t_{0}\right)=1$ here).

Although this example looks artificial, it is somewhat typical. Let $R_{0} \in \mathbb{Z}[X, Y, Z]$ be homogeneous of degree $d$ defining a smooth curve over $\mathbb{Q}$, but a singular curve modulo some prime $p$. One expects this singular curve to have a single rational node, which after a linear change of variable is $(0: 0: 1)$. So, after this change of variable, $R_{0}=P_{0}+p(a X+b Y) Z^{d-1}+$ $p c Z^{d}$ for some $a, b, c \in \mathbb{Z}$ and $P_{0}(X, Y, Z)$ homogeneous of degree $d$ with no term in $Z^{d-1}$ or $Z^{d}$. One expects the curve defined by the polynomial $P_{0}$ to have again just the single node $(0: 0: 1)$. One then computes the local factor at $p$ using the lift $P_{0}$ of $R_{0} \bmod p$ as in Example 5.5.

In Example 5.5, if we instead consider the sextic curve with the single node defined by

$$
P_{0}:=X^{6}+Y^{6}+3 Y^{4} Z^{2}+\left(2 X^{3}+X^{2} Y\right) Z^{3}+\left(X^{2}+Y^{2}\right) Z^{4}
$$


then the monodromy filtration is the same, and taking $p:=31$ we find that the reverse characteristic polynomial of the Frobenius acting on the kernel of monodromy is

$$
\begin{aligned}
Q(T)= & (1+T)\left(1+14 T^{2}+10 T^{3}+82 T^{4}-210 T^{5}-8408 T^{6}+51303 T^{7}\right. \\
& +698429 T^{8}+1580279 T^{9}+21651299 T^{10}+49302183 T^{11}-250482728 T^{12} \\
& -193939410 T^{13}+2347590382 T^{14}+8875036810 T^{15} \\
& \left.+385176597554 T^{16}+26439622160671 T^{18}\right) .
\end{aligned}
$$

This computation took around 285 hours and 14.7 GB RAM. It was too large for the author to fully check by naive point counting and illustrates the usefulness of the method, assuming Conjecture 4.11, for computing zeta functions of nodal plane curves.

EXAmple 5.7. Consider the smooth curve of genus four in $\mathbb{P}^{3}$ defined as the intersection

$$
\begin{gathered}
T^{2}+X^{2}+Y^{2}+Z^{2}=0, \\
T^{3}+2 T X Z+X^{3}-X^{2} Y+Y^{3}+Z^{3}=0 .
\end{gathered}
$$

Eliminating $T$, we obtain a birational curve in $\mathbb{P}^{2}$ of degree six with six nodes, defined by the polynomial

$$
\begin{aligned}
P_{0}:= & X^{6}-X^{5} Y-2 X^{5} Z+2 X^{4} Y^{2}+\frac{7}{2} X^{4} Z^{2}+X^{3} Y^{3}-4 X^{3} Y^{2} Z-3 X^{3} Z^{3} \\
& +\frac{1}{2} X^{2} Y^{4}+5 X^{2} Y^{2} Z^{2}-X^{2} Y Z^{3}+\frac{7}{2} X^{2} Z^{4}-2 X Y^{4} Z-4 X Y^{2} Z^{3} \\
& -2 X Z^{5}+Y^{6}+\frac{3}{2} Y^{4} Z^{2}+Y^{3} Z^{3}+\frac{3}{2} Y^{2} Z^{4}+Z^{6} .
\end{aligned}
$$

Consider now the degeneration (5.1) to this six-nodal sextic defined by taking $P_{1}:=X^{6}+Y^{6}+$ $Z^{6}$. Here $e=1$ and no pull back on the base gives non-trivial nilpotent monodromy $\mathcal{N}_{0}$. We have $\mathcal{N}_{0}^{2}=0$ and the monodromy filtration (5.2) consists of spaces of dimensions $0,6,14,20$, respectively. For $p:=7$, the reverse characteristic polynomial of the Frobenius $\mathcal{F}_{0}$ acting on $\operatorname{Ker}\left(\mathcal{N}_{0}\right)$ is

$$
\begin{aligned}
Q(T):= & \left(1-T^{2}+T^{4}-T^{6}\right) \\
& \times\left(1+2 T+11 T^{2}+40 T^{3}+72 T^{4}+280 T^{5}+539 T^{6}+686 T^{7}+2401 T^{8}\right) .
\end{aligned}
$$

The fibre $X_{0}$ is semistable with smooth total space, so we have a semistable degeneration. Indeed, $X_{1,0}:=X_{0}$ has zeta function $Q(T) /(1-T)(1-7 T)$, which supports Conjecture 4.11. The zeta function of the original genus four curve in $\mathbb{P}_{\mathbb{F}_{7}}^{3}$ has numerator the degree eight weight $\frac{1}{2}$ with respect to 7 factor of $Q(T)$.

5.2.2. Examples illustrating our construction. The next examples are primarily illustrative, although in each case we verified that the weight-monodromy conjecture held for our construction (§5.1.2). It would be interesting to compute a 'semistable limit' for the degeneration in each case. Note that for the surfaces considered, cyclotomic factors in $Q(T / p)$ should correspond to algebraic curves on the 'semistable limit', by the philosophy of the Tate conjecture if not the conjecture itself.

EXAMPLE 5.8. Consider the degeneration (5.1) of a diagonal quintic to a non-reduced union of lines defined by taking $P_{1}:=X^{5}+Y^{5}+Z^{5}$ and

$$
P_{0}:=X Y Z^{3} \text {. }
$$

Here $e=3$ and pulling back the base via $s^{3}:=t$ gives non-trivial nilpotent monodromy $\mathcal{N}_{0}$. We have $\mathcal{N}_{0}^{2}=0$ and the monodromy filtration (5.2) consists of spaces of dimensions $0,1,11,12$, respectively. Note that in this example the connection matrix had a double pole at $t=0$ with respect to the initial Dwork basis (see Corollary 2.8) and so a cyclic vector was used to obtain 
a new basis so that the connection matrix had only a simple pole (cf. Theorem 2.1). For the prime $p:=31$, the reverse characteristic polynomial of the Frobenius $\mathcal{F}_{0}$ acting on $\operatorname{Ker}\left(\mathcal{N}_{0}\right)$ is

$$
Q(T)=(1-T)\left(1+4 T+31 T^{2}\right)\left(1+8 T+33 T^{2}+248 T^{3}+961 T^{4}\right)^{2} .
$$

The zeta function of the original singular fibre $X_{0}$ is $1 /(1-31 T)^{3}$.

EXAMPLE 5.9. Consider the degeneration (5.1) of a diagonal quartic surface to a quartic surface with a pair of ordinary double points $\left(( \pm i: 1: 0: 0)\right.$, where $\left.i^{2}=-1\right)$ defined by taking $P_{1}:=X^{4}+Y^{4}+Z^{4}+W^{4}$ and

$$
P_{0}:=X^{4}+Y^{4}+Z^{4}+W^{4}+2 X^{2} Y^{2}+2 X Y Z W+2 Z W^{3} .
$$

Here $e=2$ and pulling back the base via $s^{2}:=t$ gives trivial monodromy; that is, $\mathcal{N}_{0}=0$. The monodromy filtration (5.3) has subspaces of dimensions $0,0,0,21,21,21$. For the prime $p:=5$, the reverse characteristic polynomial $Q(T)$ of the Frobenius $\mathcal{F}_{0}$ on $\mathcal{H}_{0}$ satisfies

$$
Q(T / 5)=(1-T)^{7}(1+T)^{6}\left(1+T^{2}\right)^{2}\left(1+T+T^{2}\right)\left(1+\frac{2}{5} T+T^{2}\right) .
$$

The polynomial $Q(T)$ is a weight 1 Weil polynomial with respect to 5 . By naive point counting the author verified that $\bmod T^{8}$ the local expansion at $T=0$ of the zeta function of the singular fibre $X_{0}$ is congruent to $(1+5 T)^{2}$ times the local expansion of $1 /(1-T)(1-5 T) Q(T)(1-$ $25 T)$. This suggests that a semistable degeneration can be constructed in which the degenerate fibre $X_{2,0}$ is $X_{0}$ blown up at the two singular points.

EXAmple 5.10. Consider the degeneration (5.1) of a diagonal quartic to a quartic surface with a pair of $A_{3}$ singularities $((0: 0: 0: 1)$ and $(-1: 0: 0: 1))$ defined by taking $P_{1}:=X^{4}+Y^{4}+Z^{4}+W^{4}$ and

$$
P_{0}:=X^{4}+Y^{4}+2 Z^{4}+W^{2}\left(X^{2}+Y^{2}\right)+2 W X^{3} .
$$

Here $e=4$ and pulling back the base via $s^{4}:=t$ gives trivial monodromy; that is, $\mathcal{N}_{0}=0$. The monodromy filtration (5.3) has subspaces of dimensions $0,0,0,21,21,21$. For the prime $p:=5$, the reverse characteristic polynomial $Q(T)$ of the Frobenius $\mathcal{F}_{0}$ on $\mathcal{H}_{0}$ satisfies

$$
Q(T / 5)=(1-T)^{5}(1+T)^{8}\left(1-\frac{8}{5} T+T^{2}\right)\left(1+T^{2}\right)^{3} .
$$

The polynomial $Q(T)$ is a weight 1 Weil polynomial with respect to 5 .

Example 5.11. Consider the degeneration (5.1) of a diagonal quartic to a quartic surface with an $A_{2}$ singularity $(0: 0: 0: 1)$ defined by $P_{1}:=X^{4}+Y^{4}+Z^{4}+W^{4}$ and

$$
P_{0}:=X^{4}+2 Y^{4}+2 Z^{4}+W^{2}\left(X^{2}+Y^{2}\right)+2 W Z^{3} .
$$

Here $e=3$ and pulling back the base via $s^{3}:=t$ gives trivial monodromy. The monodromy filtration (5.3) has subspaces of dimensions $0,0,0,21,21,21$. For the prime $p:=13$, the reverse characteristic polynomial $Q(T)$ of the Frobenius $\mathcal{F}_{0}$ on $\mathcal{H}_{0}$ satisfies

$$
\begin{aligned}
Q(T / 13)= & (1-T)^{2}(1+T)^{3}\left(1-T+T^{2}\right)\left(1+T+T^{2}\right)^{2}\left(1-T^{2}+T^{4}\right) \\
& \times\left(1-\frac{2}{13} T+\frac{16}{13} T^{2}-\frac{6}{13} T^{3}+\frac{16}{13} T^{4}-\frac{2}{13} T^{5}+T^{6}\right) .
\end{aligned}
$$

The polynomial $Q(T)$ is a weight 1 Weil polynomial with respect to 13 .

Example 5.12. Consider the degeneration (5.1) of a diagonal quartic to the 'Roman surface' defined by taking $P_{1}:=X^{4}+Y^{4}+Z^{4}+W^{4}$ and

$$
P_{0}:=X^{2} Y^{2}+X^{2} Z^{2}+Y^{2} Z^{2}+2 X Y Z W \text {. }
$$


Here $e=2$ and pulling back the base via $s^{2}:=t$ gives non-trivial nilpotent monodromy; the monodromy matrix $\mathcal{N}_{0}$ is such that $\mathcal{N}_{0}^{2} \neq 0$ but $\mathcal{N}_{0}^{3}=0$. The monodromy filtration (5.3) has subspaces of dimensions $0,1,1,20,20,21$. For the prime $p:=13$, the reverse characteristic polynomial $Q(T)$ of the Frobenius $\mathcal{F}_{0}$ on $\mathcal{H}_{0}$ is

$$
Q(T)=(1-169 T)(1-13 T)^{7}(1+13 T)^{12}(1-T) .
$$

Note that $\operatorname{Ker}\left(\mathcal{N}_{0}\right)$ is not a subspace in the monodromy filtration, and $\operatorname{det}\left(1-T \mathcal{F}_{0} \mid \operatorname{Ker}\left(\mathcal{N}_{0}\right)\right)=$ $(1-13 T)^{6}(1+13 T)^{12}(1-T)$.

Example 5.13. Consider the degeneration (5.1) of a diagonal quartic to a cone over a smooth quartic curve defined by taking $P_{1}:=X^{4}+Y^{4}+Z^{4}+W^{4}$ and

$$
P_{0}:=X^{4}+3 X^{3} Y+X Y Z^{2}+Y^{4}+2 Y^{3} Z+Z^{4} .
$$

Here $e=4$ and pulling back the base via $s^{4}:=t$ gives trivial monodromy. The monodromy filtration (5.3) has subspaces of dimensions $0,0,0,21,21,21$. For the prime $p:=13$, the reverse characteristic polynomial $Q(T)$ of the Frobenius $\mathcal{F}_{0}$ on $\mathcal{H}_{0}$ is such that

$$
\begin{aligned}
Q(T / 13)= & (1-T)^{3}(1+T)^{2}\left(1+T^{2}\right)\left(1+\frac{16}{13} T+\frac{21}{13} T^{2}+\frac{16}{13} T^{3}+\frac{15}{13} T^{4}+\frac{16}{13} T^{5}+\frac{19}{13} T^{6}\right. \\
& \left.+\frac{24}{13} T^{7}+\frac{19}{13} T^{8}+\frac{16}{13} T^{9}+\frac{15}{13} T^{10}+\frac{16}{13} T^{11}+\frac{21}{13} T^{12}+\frac{16}{13} T^{13}+T^{14}\right) .
\end{aligned}
$$

The polynomial $Q(T)$ is a weight 1 Weil polynomial with respect to 13 . The zeta function of the fibre $X_{0}$ is $R(13 T) /(1-T)(1-13 T)(1-169 T)$, where $R(T):=1+2 T+11 T^{2}-24 T^{3}+$ $143 T^{4}+338 T^{5}+13^{3} T^{6}$ is the numerator of the zeta function of the smooth quartic curve.

EXAmPle 5.14. Consider the degeneration (5.1) of a diagonal quartic to an irreducible quartic surface with a one-dimensional singular locus defined by taking $P_{1}:=X^{4}+Y^{4}+Z^{4}+$ $W^{4}$ and

$$
P_{0}:=X^{2} Y^{2}+Y^{2} W^{2}+X^{2} Z^{2}+Y^{2} W^{2}+X^{2} W^{2}+Y^{2} Z^{2}+2 X Y Z W .
$$

Here $e=2$ and pulling back the base via $s^{2}:=t$ gives a non-trivial monodromy matrix $\mathcal{N}_{0}$ with $\mathcal{N}_{0}^{2}=0$. The monodromy filtration (5.3) has subspaces of dimensions $0,0,2,19,21,21$. For the prime $p:=13$, the reverse characteristic polynomial $Q(T)$ of the Frobenius $\mathcal{F}_{0}$ on $\mathcal{H}_{0}$ is

$$
Q(T)=(1-13 T)^{7}(1+13 T)^{10}\left(1+2 T+13 T^{2}\right)\left(1+26 T+2197 T^{2}\right) .
$$

The last two factors here are weight $\frac{1}{2}$ and weight $\frac{3}{2}$ Weil polynomials with respect to 13 , respectively.

ExAmple 5.15. Consider the degeneration (5.1) of a diagonal cubic threefold to a singular threefold containing a pencil of planes defined by taking $P_{1}:=X^{3}+Y^{3}+Z^{3}+U^{3}+V^{3}$ and

$$
P_{0}:=Z X^{2}+U X Y+V Y^{2} .
$$

Here $e=2$ and pulling back the base via $s^{2}:=t$ gives trivial monodromy. So, $\mathcal{N}_{0}=0$ and the monodromy filtration (5.4) has subspaces of dimensions $0,0,0,0,10,10,10,10$. For the prime $p:=19$, the reverse characteristic polynomial $Q(T)$ of the Frobenius $\mathcal{F}_{0}$ on $\mathcal{H}_{0}$ is such that

$$
Q(T / 19)=\left(1-2 T+19 T^{2}\right)^{2}\left(1+8 T+19 T^{2}\right)^{2}\left(1-T+19 T^{2}\right) .
$$

Thus, $Q(T)$ is a weight $\frac{3}{2}$ Weil polynomial with respect to 19 .

Acknowledgements. The author would like to thank Bruno Chiarellotto, Richard Crew, Kiran Kedlaya, Bernard Le Stum, Damiano Testa, Nobuo Tsuzuki and the referee. 


\section{References}

1. T. G. Аввот, K. S. Kedlaya and D. Roe, 'Bounding Picard numbers of surfaces using p-adic cohomology', Arithmetic, geometry and coding theory (AGCT 2005), Séminaires et Congrès 21 (eds F. Rodier and S. Vladut; Societé Mathématique de France, 2009) 125-159.

2. B. Chiarellotto, 'Rigid cohomology and invariant cycles for a semistable log scheme', Duke Math. J. 97 (1999) no. 1, 155-169.

3. B. Chiarellotto and B. Le Stum, 'A comparison theorem for weights', J. Reine Angew. Math. 546 (2002) $159-176$.

4. G. Christol and Z. Mebkhout, 'Sur le théorème de l'indice des équations diffèrentielles $p$-adiques II', Ann. of Math. (2) 146 (1997) no. 2, 345-410.

5. D. A. Cox and S. KATz, Mirror symmetry and algebraic geometry, Mathematical Surveys and Monographs 68 (American Mathematical Society, Providence, RI, 1999).

6. R. CREw, 'Finiteness theorems for the cohomology of an overconvergent isocrystal on a curve', Ann. Sci. École Norm. Sup. 31 (1998) no. 6, 717-763.

7. A. Dimca, 'On the de Rham cohomology of a hypersurface complement', Amer. J. Math. 113 (1991) no. 4, $763-771$.

8. B. Dwork, 'On the rationality of the zeta function of an algebraic variety', Amer. J. Math. 82 (1960) 631-648.

9. B. Dwork, G. Gerotto and F. J. Sullivan, An introduction to G-functions, Annals of Mathematics Studies 133 (Princeton University Press, Princeton, NJ, 1994).

10. R. Gerkman, 'Relative rigid cohomology and deformation of hypersurfaces', Int. Math. Res. Pap. IMRP (2007) no. 1, 67, Art. ID rpm003.

11. J. Harris and I. Morrison, Moduli of curves, Graduate Texts in Mathematics 187 (Springer, New York, 1998).

12. L. Illusie, 'Autour de théoreme de monodromie locale, Périodes p-adique (Bures-sur-Yvette, 1988)', Astérisque 223 (1994) 9-57.

13. N. M. KATZ, 'Nilpotent connections and the monodromy theorem: applications of a result of Turrittin', Publ. Math. Inst. Hautes Études Sci. 39 (1970) 175-232.

14. N. M. KATZ and T. ODA, 'On the differentiation of de Rham cohomology classes with respect to parameters', J. Math. Kyoto Univ. 8 (1968) 199-213.

15. K. S. Kedlaya, 'Effective $p$-adic cohomology for cyclic cubic threefolds'. Available at www.mit.edu/ kedlaya/papers/.

16. K. S. Kedlaya, 'Semistable reduction for overconvergent $F$-isocrystals on a curve', Math. Res. Lett. 10 (2003) 151-159.

17. K. S. Kedlaya, $p$-adic differential equations (Cambridge University Press, Cambridge, 2010).

18. R. Kloosterman, 'Point counting on singular hypersurfaces', Algorithmic number theory: ANTS VIII, Lecture Notes in Computer Science 5011 (eds A. J. van der Poorten and A. Stein; Springer, Berlin, 2008) 327-341.

19. J. Kollár, Lectures on resolution of singularities, Annals of Mathematics Studies 166 (Princeton University Press, Princeton, NJ, 2007).

20. A. Landman, 'On the Picard-Lefschetz transformation for algebraic manifolds acquiring general singularities', Trans. Amer. Math. Soc. 181 (1973) 89-126.

21. S. LAng, Algebra (revised third edition), Graduate Texts in Mathematics 211 (Springer, Berlin, 2002).

22. A. G. B. LAUder, 'Counting solutions to equations in many variables over finite fields', Found. Comput. Math. 4 (2004) no. 3, 221-267.

23. A. G. B. LAuder, 'A recursive method for computing zeta functions of varieties', LMS J. Comput. Math. 9 (2006) 222-269 (electronic).

24. B. Le Stum, Rigid cohomology, Cambridge Tracts in Mathematics 172 (Cambridge University Press, Cambridge, 2007).

25. B. Le Stum, 'The overconvergent site', Mém. Soc. Math. Fr. (N.S.), to appear. Preprint (2011), available at perso.univ-rennes1.fr/bernard.le-stum/.

26. F. S. Macaulay, The algebraic theory of modular systems (Cambridge University Press, Cambridge, 1916).

27. B. Mazur, 'Frobenius and the Hodge filtration', Bull. Amer. Math. Soc. 78 (1972) 653-667.

28. Y. MiedA, 'The Picard-Lefschetz formula for p-adic cohomology', Math. Z. 257 (2007) 403-425.

29. D. S. Morrison, 'The Clemens-Schmidt exact sequence and applications', Topics in transcendental algebraic geometry, Annals of Mathematics Studies 106 (ed. P. Griffiths; Princeton University Press, Princeton, NJ, 1984) 101-119.

30. D. Mumford, 'Semi-stable reduction', Toroidal embeddings I, Lecture Notes in Mathematics 339 (eds G. Kempf, F. Knudsen, D. Mumford and B. Saint-Donat; Springer, Berlin, 1973) 53-108.

31. S. PAncratz, 'Computing Gauss-Manin connections for families of projective hypersurfaces', D. Phil. Transfer of Status Thesis, University of Oxford, 2009. Available at www.pancratz.org.

32. C. A. M. Peters and J. H. M. Steenbrink, Mixed Hodge structures (Springer, Berlin, 2008). 
33. D. Petrequin, 'Classes de Chern et classes de cycles en cohomologie rigide', Bull. Soc. Math. France 131 (2003) no. 1, 59-121.

34. M. VAN Der Put and M. Singer, Galois theory of linear differential equations (Springer, Berlin, 2003).

\author{
Alan G. B. Lauder \\ Mathematical Institute \\ University of Oxford \\ 24-29 St Giles \\ Oxford OX1 3LB \\ United Kingdom \\ lauder@maths.ox.ac.uk
}

